\title{
Level Zero Fundamental Representations over Quantized Affine Algebras and Demazure Modules
}

By

\author{
Masaki KashiwARA*
}

\begin{abstract}
Let $W\left(\varpi_{k}\right)$ be the finite-dimensional irreducible module over a quantized affine algebra $U_{q}^{\prime}(\mathfrak{g})$ with the fundamental weight $\varpi_{k}$ as an extremal weight. We show that its crystal $B\left(W\left(\varpi_{k}\right)\right)$ is isomorphic to the Demazure crystal $B^{-}\left(-\Lambda_{0}+\varpi_{k}\right)$. This is derived from the following general result: for a dominant integral weight $\lambda$ and an integral weight $\mu$, there exists a unique homomorphism $U_{q}^{-}(\mathfrak{g})\left(u_{\lambda} \otimes u_{\mu}\right) \rightarrow V(\lambda+\mu)$ that sends $u_{\lambda} \otimes u_{\mu}$ to $u_{\lambda+\mu}$. Here $V(\lambda)$ is the extremal weight module with $\lambda$ as an extremal weight, and $u_{\lambda} \in V(\lambda)$ is the extremal weight vector of weight $\lambda$.
\end{abstract}

\section{$\S 1$. Introduction}

The finite-dimensional representations of quantized affine algebras $U_{q}^{\prime}(\mathfrak{g})$ are extensively studied in connection with exactly solvable models. It is expected that there exists a "good" finite-dimensional $U_{q}^{\prime}(\mathfrak{g})$-module $W\left(m \varpi_{k}\right)$ with a multiple $m \varpi_{k}$ of a fundamental weight $\varpi_{k}$ as an extremal weight. This module is good in the sense that it is irreducible and it has a crystal base and moreover a global basis.

In the untwisted case, its conjectural character formula is given by KirillovReshetikhin ([17], see also [16]), and its conjectural fusion construction is given by Kuniba-Nakanishi-Suzuki ([18]). It is proved by Nakajima ([22]) that the

Communicated by T. Kawai. Received January 27, 2004. Revised August 10, 2004.

1991 Mathematics Subject Classification. Primary 20G05; Secondary 17B37.

Key words: Crystal bases, extremal modules, fundamental representations, Demazure modules.

* Research Institute for Mathematical Sciences, Kyoto University, Kyoto 606-8502, Japan This research is partially supported by Grant-in-Aid for Scientific Research (B1)13440006, Japan Society for the Promotion of Science. 
fusion construction gives irreducible modules with the expected character in the simply laced case, and by Chari ([3]) in some cases.

It is also expected that any "good" finite-dimensional $U_{q}^{\prime}(\mathfrak{g})$-module is a tensor product of modules of the above type.

It is also conjectured in $[4,5]$ that the $U_{q}^{\prime}(\mathfrak{g})$-modules $W\left(m \varpi_{k}\right)$ has a perfect crystal of level $\ell$ if and only if $m=\ell c_{k}^{\vee}\left(c_{k}^{\vee}:=\max \left(1,2 /\left(\alpha_{k}, \alpha_{k}\right)\right)\right)$. Moreover it is conjectured that the crystal base $B\left(W\left(\ell c_{k}^{\vee} \varpi_{k}\right)\right)$ is isomorphic to the Demazure crystal $B^{-}\left(-\ell \Lambda_{0}+\ell c_{k}^{\vee} \varpi_{k}\right)$ if we forget the 0 -arrows. Here, for an integral weight $\lambda, B^{ \pm}(\lambda)$ denotes the crystal for the $U_{q}^{ \pm}(\mathfrak{g})$-module generated by the extremal vector with weight $\lambda$. They are proved in certain cases $([7,8])$. More general relations of perfect crystals and Demazure crystals are discussed in $[6]$.

In this paper we show that $B\left(W\left(\varpi_{k}\right)\right)$ is isomorphic to the Demazure crystal $B^{-}\left(-\Lambda_{0}+\varpi_{k}\right)$, or equivalently $B\left(W\left(-\varpi_{k}\right)\right)$ is isomorphic to the Demazure crystal $B^{+}\left(\Lambda_{0}-\varpi_{k}\right)$ (Corollary 4.8 ).

The main ingredient is the following theorem, which the author started to study in order to answer a question raised by Miwa et al:

Theorem 3.3. Let $U_{q}^{-}(\mathfrak{g})$ be a quantized affine algebra. Let $\lambda \in P^{+}$be a dominant integral weight and $\mu \in P$ an integral weight. Then there exists a unique homomorphism $V(\lambda) \otimes V(\mu) \supset U_{q}^{-}(\mathfrak{g})\left(u_{\lambda} \otimes u_{\mu}\right) \longrightarrow V(\lambda+\mu)$ that sends $u_{\lambda} \otimes u_{\mu}$ to $u_{\lambda+\mu}$. Moreover this morphism is compatible with global bases.

Here $V(\lambda)$ is the extremal weight module with $\lambda$ as an extremal weight, and $u_{\lambda} \in V(\lambda)$ is the extremal weight vector of weight $\lambda$.

\section{§2. Review on Crystal Bases and Global Bases}

In this section, we shall review briefly the quantized universal enveloping algebras and crystal bases. We refer the reader to $[9,10,13,14,15,19]$.

\section{§2.1. Quantized universal enveloping algebras}

We shall define the quantized universal enveloping algebra $U_{q}(\mathfrak{g})$. Assume that we are given the following data.

$$
\begin{aligned}
& P \text { : a free } \mathbb{Z} \text {-module (called a weight lattice), } \\
& I: \text { an index set (for simple roots), }
\end{aligned}
$$




$$
\begin{aligned}
& \alpha_{i} \in P \text { for } i \in I \text { (called a simple root), } \\
& h_{i} \in P^{*}:=\operatorname{Hom}_{\mathbb{Z}}(P, \mathbb{Z}) \text { (called a simple coroot), } \\
& (\cdot, \cdot): P \times P \rightarrow \mathbb{Q} \text { a bilinear symmetric form. }
\end{aligned}
$$

We shall denote by $\langle\cdot, \cdot\rangle: P^{*} \times P \rightarrow \mathbb{Z}$ the canonical pairing.

The data above are assumed to satisfy the following axioms.

$$
\begin{aligned}
& \left(\alpha_{i}, \alpha_{i}\right)>0 \text { for any } i \in I, \\
& \left(\alpha_{i}, \alpha_{j}\right) \leqslant 0 \text { for any } i, j \in I \text { with } i \neq j, \\
& \left\langle h_{i}, \lambda\right\rangle=\frac{2\left(\alpha_{i}, \lambda\right)}{\left(\alpha_{i}, \alpha_{i}\right)} \quad \text { for any } i \in I \text { and } \lambda \in P .
\end{aligned}
$$

Let us take a positive integer $d$ such that $\left(\alpha_{i}, \alpha_{i}\right) / 2 \in \mathbb{Z} d^{-1}$ for any $i \in I$. Now let $q$ be an indeterminate and set

$$
K=\mathbb{Q}\left(q_{\mathrm{s}}\right) \text { where } q_{\mathrm{s}}=q^{1 / d} .
$$

We define its subrings $\mathbf{A}_{0}, \mathbf{A}_{\infty}$ and $\mathbf{A}$ as follows.

$$
\begin{aligned}
\mathbf{A}_{0} & =\left\{f / g ; f, g \in \mathbb{Q}\left[q_{\mathrm{s}}\right], g(0) \neq 0\right\}, \\
\mathbf{A}_{\infty} & =\left\{f / g ; f, g \in \mathbb{Q}\left[q_{\mathrm{s}}{ }^{-1}\right], g(0) \neq 0\right\}, \\
\mathbf{A} & =\mathbb{Q}\left[q_{\mathrm{s}}, q_{\mathrm{s}}{ }^{-1}\right] .
\end{aligned}
$$

Definition 2.1. $\quad$ The quantized universal enveloping algebra $U_{q}(\mathfrak{g})$ is the algebra over $K$ generated by the symbols $e_{i}, f_{i}(i \in I)$ and $q(h)\left(h \in d^{-1} P^{*}\right)$ with the following defining relations.

(1) $q\left(h_{1}\right) q\left(h_{2}\right)=q\left(h_{1}+h_{2}\right)$ for $h_{1}, h_{2} \in d^{-1} P^{*}$, and $q(h)=1$ for $h=0$.

(2) $q(h) e_{i} q(h)^{-1}=q^{\left\langle h, \alpha_{i}\right\rangle} e_{i}$ and $q(h) f_{i} q(h)^{-1}=q^{-\left\langle h, \alpha_{i}\right\rangle} f_{i}$ for any $i \in I$ and $h \in d^{-1} P^{*}$.

(3) $\left[e_{i}, f_{j}\right]=\delta_{i j} \frac{t_{i}-t_{i}^{-1}}{q_{i}-q_{i}^{-1}}$ for $i, j \in I$. Here $q_{i}=q^{\left(\alpha_{i}, \alpha_{i}\right) / 2}$ and $t_{i}=q\left(\frac{\left(\alpha_{i}, \alpha_{i}\right)}{2} h_{i}\right)$.

(4) (Serre relation) For $i \neq j$,

$$
\sum_{k=0}^{b}(-1)^{k} e_{i}^{(k)} e_{j} e_{i}^{(b-k)}=\sum_{k=0}^{b}(-1)^{k} f_{i}^{(k)} f_{j} f_{i}^{(b-k)}=0 .
$$

Here $b=1-\left\langle h_{i}, \alpha_{j}\right\rangle$ and

$$
\begin{aligned}
e_{i}^{(k)}=e_{i}^{k} /[k]_{i} !, f_{i}^{(k)} & =f_{i}^{k} /[k]_{i} ! \\
{[k]_{i}=\left(q_{i}^{k}-q_{i}^{-k}\right) /\left(q_{i}-q_{i}^{-1}\right),[k]_{i} ! } & =[1]_{i} \cdots[k]_{i} .
\end{aligned}
$$


For $i \in I$, we denote by $U_{q}^{-}(\mathfrak{g})_{i}$ the subalgebra of $U_{q}^{-}(\mathfrak{g})$ generated by $e_{i}$, $f_{i}$ and $q(h)\left(h \in d^{-1} P^{*}\right)$.

Let us denote by $W$ the Weyl group, the subgroup of $G L(P)$ generated by the simple reflections $s_{i}: s_{i}(\lambda)=\lambda-\left\langle h_{i}, \lambda\right\rangle \alpha_{i}$.

Let $\Delta \subset Q:=\sum_{i} \mathbb{Z} \alpha_{i}$ be the set of roots. Let $\Delta^{ \pm}:=\Delta \cap Q_{ \pm}$be the set of positive and negative roots, respectively. Here $Q_{ \pm}:= \pm \sum_{i} \mathbb{Z}_{\geqslant 0} \alpha_{i}$. Let $\Delta^{\text {re }}$ be the set of real roots, and set $\Delta_{ \pm}^{\mathrm{re}}:=\Delta_{ \pm} \cap \Delta^{\mathrm{re}}$.

\section{$\S 2.2$. Braid group action on integrable modules}

The $q$-analogue of the action of the Weyl group is introduced in [19, 23]. We define a $q$-analog of the exponential function by

$$
\exp _{q}(x)=\sum_{n=0}^{\infty} \frac{q^{n(n-1) / 2} x^{n}}{[n] !} .
$$

This satisfies the following equations:

$$
\begin{aligned}
& \exp _{q}(x) \exp _{q}(y)=\exp _{q}(x+y) \quad \text { if } x y=q^{2} y x, \\
& \exp _{q}(x) \exp _{q^{-1}}(y)=\sum_{n=0}^{\infty} \frac{1}{[n] !} \prod_{\nu=0}^{n-1}\left(q^{\nu} x+q^{-\nu} y\right) \quad \text { if }[x, y]=0, \\
& \exp _{q}(x) \exp _{q^{-1}}(-x)=1, \\
& \exp _{q}(x)=\left(1+\left(1-q^{2}\right) x\right) \exp _{q}\left(q^{2} x\right), \\
& \exp _{q}(x)=\prod_{n=0}^{\infty}\left(1+q^{2 n}\left(1-q^{2}\right) x\right) \text { for }|q|<1,
\end{aligned}
$$

For $i \in I$, we set

$$
\begin{aligned}
S_{i} & =\exp _{q_{i}^{-1}}\left(q_{i}^{-1} e_{i} t_{i}^{-1}\right) \exp _{q_{i}^{-1}}\left(-f_{i}\right) \exp _{q_{i}^{-1}}\left(q_{i} e_{i} t_{i}\right) q_{i}^{h_{i}\left(h_{i}+1\right) / 2} \\
& =\exp _{q_{i}^{-1}}\left(-q_{i}^{-1} f_{i} t_{i}\right) \exp _{q_{i}^{-1}}\left(e_{i}\right) \exp _{q_{i}^{-1}}\left(-q_{i} f_{i} t_{i}^{-1}\right) q_{i}^{h_{i}\left(h_{i}+1\right) / 2} .
\end{aligned}
$$

We regard $S_{i}$ as an endomorphism of integrable $U_{q}(\mathfrak{g})$-modules, and $q_{i}^{h_{i}\left(h_{i}+1\right) / 2}$ acts on the weight space of weight $\lambda$ by the multiplication of $q_{i}^{\left\langle h_{i}, \lambda\right\rangle\left(\left\langle h_{i}, \lambda\right\rangle+1\right) / 2}$.

On the $(l+1)$-dimensional irreducible representation of $U_{q}(\mathfrak{g})_{i}$ with a highest weight vector $u_{0}^{(l)}$ and $u_{k}^{(l)}=f_{i}^{(k)} u_{0}^{(l)}$,

$$
S_{i}\left(u_{k}^{(l)}\right)=(-1)^{l-k} q_{i}^{(l-k)(k+1)} u_{l-k}^{(l)},
$$

Hence, $S_{i}$ sends the weight space of weight $\lambda$ to the weight space of weight $s_{i} \lambda$. By the above formula, we have

$$
S_{i} u_{l}^{(l)}=u_{0}^{(l)} \quad \text { and } \quad S_{i} u_{0}^{(l)}=\left(-q_{i}\right)^{l} u_{l}^{(l)} .
$$


Since $\left\{S_{i}\right\}$ satisfies the braid relations, we can extend the actions of $S_{i}$ on integrable modules to the action of the braid group by

$$
\begin{aligned}
S_{w w^{\prime}} & =S_{w} \circ S_{w^{\prime}} \quad \text { if } l\left(w w^{\prime}\right)=l(w)+l\left(w^{\prime}\right), \\
S_{s_{i}} & =S_{i} .
\end{aligned}
$$

\section{§2.3. Braid group action on $U_{q}^{-}(\mathfrak{g})$}

We define the ring automorphism $T_{i}$ of $U_{q}(\mathfrak{g})$ by

$$
\begin{aligned}
T_{i}(q) & =q \\
T_{i}(q(h)) & =q\left(s_{i} h\right), \\
T_{i}\left(e_{i}\right) & =-f_{i} t_{i}, \\
T_{i}\left(f_{i}\right)= & -t_{i}^{-1} e_{i}, \\
T_{i}\left(e_{j}\right)= & \sum_{k=0}^{-\left\langle h_{i}, \alpha_{j}\right\rangle}(-1)^{k} q_{i}^{-k} e_{i}^{\left(-\left\langle h_{i}, \alpha_{j}\right\rangle-k\right)} e_{j} e_{i}^{(k)}, \\
T_{i}\left(f_{j}\right)= & -\left\langle\sum_{k=0}^{\left., \alpha_{j}\right\rangle}(-1)^{k} q_{i}^{k} f_{i}^{(k)} f_{j} f_{i}^{\left(-\left\langle h_{i}, \alpha_{j}\right\rangle-k\right)} \text { for } i \neq j .\right.
\end{aligned}
$$

Then it is well-defined, and it satisfies

$$
T_{i}(P) u=S_{i} P S_{i}^{-1} u
$$

for any $P \in U_{q}(\mathfrak{g})$ and any element $u$ of an integrable $U_{q}(\mathfrak{g})$-module.

The operator $T_{i}$ is invertible and its inverse is given as follows.

$$
\begin{aligned}
T_{i}^{-1}(q(h)) & =q\left(s_{i} h\right), \\
T_{i}^{-1}\left(e_{i}\right) & =-t_{i}^{-1} f_{i}, \\
T_{i}^{-1}\left(f_{i}\right) & =-e_{i} t_{i}, \\
T_{i}^{-1}\left(e_{j}\right) & =\sum_{k=0}^{-\left\langle h_{i}, \alpha_{j}\right\rangle}(-1)^{k} q_{i}^{-k} e_{i}^{(k)} e_{j} e_{i}^{\left(-\left\langle h_{i}, \alpha_{j}\right\rangle-k\right)}, \\
T_{i}^{-1}\left(f_{j}\right) & =\sum_{k=0}^{-\left\langle h_{i}, \alpha_{j}\right\rangle}(-1)^{k} q_{i}^{k} f_{i}^{\left(-\left\langle h_{i}, \alpha_{j}\right\rangle-k\right)} f_{j} f_{i}^{(k)} .
\end{aligned}
$$

We can extend the action $T_{i}$ to the action of the braid group by

$$
\begin{aligned}
T_{w w^{\prime}} & =T_{w} \circ T_{w^{\prime}} \quad \text { if } l\left(w w^{\prime}\right)=l(w)+l\left(w^{\prime}\right), \\
T_{s_{i}} & =T_{i} .
\end{aligned}
$$

The following proposition is proved in [19]. 
Proposition 2.2. For $w \in W$ and $i, j \in I$ such that $w \alpha_{i}=\alpha_{j}$, we have

$$
T_{w} e_{i}=T_{w^{-1}}^{-1} e_{i}=e_{j} \quad \text { and } \quad T_{w} f_{i}=T_{w^{-1}}^{-1} f_{i}=f_{j} .
$$

\section{§2.4. Crystals}

We shall not review the notion of crystals, but refer the reader to $[9,10$, $13,15]$. For a subset $J$ of $I$, let us denote by $U_{q}\left(\mathfrak{g}_{J}\right)$ the subalgebra of $U_{q}^{-}(\mathfrak{g})$ generated by $e_{j}, f_{j}(j \in J)$ and $q(h)\left(h \in d^{-1} P^{*}\right)$. We say that a crystal $B$ over $U_{q}^{-}(\mathfrak{g})$ is a regular crystal if, for any $J \subset I$ of finite-dimensional type, $B$ is, as a crystal over $U_{q}\left(\mathfrak{g}_{J}\right)$, isomorphic to a crystal base associated with an integrable $U_{q}\left(\mathfrak{g}_{J}\right)$-module.

By [13], the Weyl group $W$ acts on any regular crystal. This action $S$ is given by

$$
S_{s_{i}} b= \begin{cases}\tilde{f}_{i}^{\left\langle h_{i}, \operatorname{wt}(b)\right\rangle} b & \text { if }\left\langle h_{i}, \operatorname{wt}(b)\right\rangle \geqslant 0, \\ \tilde{e}_{i}^{-\left\langle h_{i}, \operatorname{wt}(b)\right\rangle} b & \text { if }\left\langle h_{i}, \operatorname{wt}(b)\right\rangle \leqslant 0 .\end{cases}
$$

Let us denote by $U_{q}^{-}(\mathfrak{g})$ (resp. $\left.U_{q}^{+}(\mathfrak{g})\right)$ the subalgebra of $U_{q}^{-}(\mathfrak{g})$ generated by the $f_{i}$ 's (resp. by the $e_{i}$ 's). Then $U_{q}^{-}(\mathfrak{g})$ has a crystal base denoted by $B(\infty)$ ([10]). A unique vector of $B(\infty)$ with weight 0 is denoted by $u_{\infty}$. Similarly $U_{q}^{+}(\mathfrak{g})$ has a crystal base denoted by $B(-\infty)$, and a unique vector of $B(-\infty)$ with weight 0 is denoted by $u_{-\infty}$.

Let $\psi$ be the ring automorphism of $U_{q}^{-}(\mathfrak{g})$ that sends $q_{\mathrm{s}}, e_{i}, f_{i}$ and $q(h)$ to $q_{\mathrm{s}}, f_{i}, e_{i}$ and $q(-h)$. It induces bijections $U_{q}^{-}(\mathfrak{g}) \stackrel{\sim}{\longrightarrow} U_{q}^{+}(\mathfrak{g})$ and $B(\infty) \stackrel{\sim}{\longrightarrow} B(-\infty)$ by which $u_{\infty}, \tilde{e}_{i}, \tilde{f}_{i}, \varepsilon_{i}, \varphi_{i}$, wt correspond to $u_{-\infty}, \tilde{f}_{i}, \tilde{e}_{i}, \varphi_{i}, \varepsilon_{i},-$ wt.

Let $\widetilde{U}_{q}(\mathfrak{g})$ be the modified quantized universal enveloping algebra $\oplus_{\lambda \in P}$ $U_{q}^{-}(\mathfrak{g}) a_{\lambda}$ (see [13]). The elements $a_{\lambda}$, the projectors to the weight $\lambda$-space, satisfy $a_{\lambda} \cdot a_{\mu}=\delta_{\lambda, \mu} a_{\lambda}$ and $a_{\lambda} P=P a_{\lambda-\mathrm{wt}(P)}$ for $P \in U_{q}^{-}(\mathfrak{g})$.

Then $\widetilde{U}_{q}(\mathfrak{g})$ has a crystal base $\left(L\left(\widetilde{U}_{q}(\mathfrak{g})\right), B\left(\widetilde{U}_{q}(\mathfrak{g})\right)\right)$. As a crystal, $B\left(\widetilde{U}_{q}(\mathfrak{g})\right)$ is regular and isomorphic to

$$
\bigsqcup_{\lambda \in P} B(\infty) \otimes T_{\lambda} \otimes B(-\infty)
$$

Here, $T_{\lambda}$ is the crystal consisting of a single element $t_{\lambda}$ with $\varepsilon_{i}\left(t_{\lambda}\right)=\varphi_{i}\left(t_{\lambda}\right)=$ $-\infty$ and $\operatorname{wt}\left(t_{\lambda}\right)=\lambda$.

Let $*$ be the anti-involution of $U_{q}^{-}(\mathfrak{g})$ that sends $q(h)$ to $q(-h)$, and $q_{\mathrm{s}}, e_{i}$, $f_{i}$ to themselves. The involution $*$ of $U_{q}^{-}(\mathfrak{g})$ induces an involution $*$ on $B(\infty)$, $B(-\infty), B\left(\widetilde{U}_{q}(\mathfrak{g})\right)$. Then $\tilde{e}_{i}^{*}=* \circ \tilde{e}_{i} \circ *$, etc. give another crystal structure 
on $B(\infty), B(-\infty), B\left(\widetilde{U}_{q}(\mathfrak{g})\right)$. We call it the star crystal structure. These two crystal structures on $B\left(\widetilde{U}_{q}(\mathfrak{g})\right)$ are compatible, and $B\left(\widetilde{U}_{q}(\mathfrak{g})\right)$ may be considered as a crystal over $\mathfrak{g} \oplus \mathfrak{g}$, which corresponds to the $U_{q}^{-}(\mathfrak{g})$-bimodule structure on $\widetilde{U}_{q}(\mathfrak{g})$. Hence, for example, $S_{w}^{*}$, the Weyl group action on $B\left(\widetilde{U}_{q}(\mathfrak{g})\right)$ with respect to the star crystal structure is a crystal automorphism of $B\left(\widetilde{U}_{q}(\mathfrak{g})\right)$ with respect to the original crystal structure. In particular, the two Weyl group actions $S_{w}$ and $S_{w^{\prime}}^{*}$ commute with each other.

\section{$\S 2.5$. Global bases}

Recall that $\mathbf{A}_{0} \subset K$ is the subring of $K$ consisting of rational functions in $q_{\mathrm{s}}$ without pole at $q_{\mathrm{s}}=0$. Let - be the automorphism of $K$ sending $q_{\mathrm{s}}$ to $q_{\mathrm{s}}{ }^{-1}$. Then $\overline{\mathbf{A}_{0}}$ coincides with the ring $\mathbf{A}_{\infty}$ of rational functions regular at $q_{\mathrm{s}}=\infty$. Set $\mathbf{A}:=\mathbb{Q}\left[q_{\mathrm{s}}, q_{\mathrm{s}}{ }^{-1}\right]$. Let $V$ be a vector space over $K, L_{0}$ an $A$-submodule of $V, L_{\infty}$ an $\mathbf{A}_{\infty}$-submodule, and $V_{\mathbf{A}}$ a $\mathbf{A}$-submodule. Set $E:=L_{0} \cap L_{\infty} \cap V_{\mathbf{A}}$.

Definition 2.3 [10]. We say that $\left(L_{0}, L_{\infty}, V_{\mathbf{A}}\right)$ is balanced if each of $L_{0}, L_{\infty}$ and $V_{\mathbf{A}}$ generates $V$ as a $K$-vector space, and if one of the following equivalent conditions is satisfied.

(i) $E \rightarrow L_{0} / q_{\mathrm{s}} L_{0}$ is an isomorphism.

(ii) $E \rightarrow L_{\infty} / q_{\mathrm{s}}{ }^{-1} L_{\infty}$ is an isomorphism.

(iii) $\left(L_{0} \cap V_{\mathbf{A}}\right) \oplus\left(q_{\mathrm{s}}{ }^{-1} L_{\infty} \cap V_{\mathbf{A}}\right) \rightarrow V_{\mathbf{A}}$ is an isomorphism.

(iv) $\mathbf{A}_{0} \otimes_{\mathbb{Q}} E \rightarrow L_{0}, \mathbf{A}_{\infty} \otimes_{\mathbb{Q}} E \rightarrow L_{\infty}, \mathbf{A} \otimes_{\mathbb{Q}} E \rightarrow V_{\mathbf{A}}$ and $K \otimes_{\mathbb{Q}} E \rightarrow V$ are isomorphisms.

Let - be the ring automorphism of $U_{q}^{-}(\mathfrak{g})$ sending $q_{\mathrm{s}}, q(h), e_{i}, f_{i}$ to $q_{\mathrm{s}}{ }^{-1}$, $q(-h), e_{i}, f_{i}$.

Let $U_{q}^{-}(\mathfrak{g})_{\mathbf{A}}$ be the A-subalgebra of $U_{q}^{-}(\mathfrak{g})$ generated by $e_{i}^{(n)}, f_{i}^{(n)}$ and $q(h)\left(h \in d^{-1} P^{*}\right)$.

Let $M$ be a $U_{q}^{-}(\mathfrak{g})$-module. Let - be an involution of $M$ satisfying $(a u)^{-}=$ $\bar{a} \bar{u}$ for any $a \in U_{q}^{-}(\mathfrak{g})$ and $u \in M$. We call in this paper such an involution a bar involution. Let $(L(M), B(M))$ be a crystal base of an integrable $U_{q}^{-}(\mathfrak{g})$-module M.

Let $M_{\mathbf{A}}$ be a $U_{q}^{-}(\mathfrak{g})_{\mathbf{A}}$-submodule of $M$ such that

$$
\left(M_{\mathbf{A}}\right)^{-}=M_{\mathbf{A}}, \text { and }(u-\bar{u}) \in\left(q_{\mathrm{s}}-1\right) M_{\mathbf{A}} \text { for every } u \in M_{\mathbf{A}} .
$$


Definition 2.4. A $U_{q}^{-}(\mathfrak{g})$-module $M$ endowed with $(L(M), B(M)$, $\left.M_{\mathbf{A}},-\right)$ as above is called with a global basis, if $\left(L(M), L(M)^{-}\right.$, $\left.M_{\mathbf{A}}\right)$ is balanced,

In such a case, let $G: L(M) / q_{\mathrm{s}} L(M) \stackrel{\sim}{\longrightarrow} E:=L(M) \cap L(M)^{-} \cap M_{\mathbf{A}}$ be the inverse of $E \stackrel{\sim}{\longrightarrow} L(M) / q_{\mathrm{s}} L(M)$. Then $\{G(b) ; b \in B(M)\}$ forms a basis of $M$. We call this basis a (lower) global basis. The global basis enjoys the following properties (see $[10,11])$ :

(i) $\overline{G(b)}=G(b)$ for any $b \in B(M)$.

(ii) For any $n \in \mathbb{Z}_{\geqslant 0},\left\{G(b) ; \varepsilon_{i}(b) \geqslant n\right\}$ is a basis of the $\mathbf{A}$-submodule $\sum_{m \geqslant n}$ $f_{i}^{(m)} M_{\mathbf{A}}$.

(iii) for any $i \in I$ and $b \in B(M)$, we have

$$
f_{i} G(b)=\left[1+\varepsilon_{i}(b)\right]_{i} G\left(\tilde{f}_{i} b\right)+\sum_{b^{\prime}} F_{b, b^{\prime}}^{i} G\left(b^{\prime}\right) .
$$

Here the sum ranges over $b^{\prime} \in B(M)$ such that $\varepsilon_{i}\left(b^{\prime}\right)>1+\varepsilon_{i}(b)$. The coefficient $F_{b, b^{\prime}}^{i}$ belongs to $q_{\mathrm{s}} q_{i}^{1-\varepsilon_{i}\left(b^{\prime}\right)} \mathbb{Q}\left[q_{\mathrm{s}}\right]$. Similarly for $e_{i} G(b)$.

Let $M$ and $N$ be $U_{q}^{-}(\mathfrak{g})$-modules with global bases. We say that a $U_{q}^{-}(\mathfrak{g})$ morphism $f: M \rightarrow N$ is compatible with global bases if it satisfies the following conditions:

(i) If $u$ is a global basis vector of $M$, then $f(u)$ is a global basis vector of $N$ or 0 .

(ii) If a pair of global basis vectors $u$ and $v$ of $M$ satisfies $f(u)=f(v) \neq 0$, then $u=v$.

These conditions are equivalent to the following set of conditions:

(a) $f$ commutes with the bar involutions.

(b) $f$ sends $L(M)$ to $L(N)$ and $M_{\mathbf{A}}$ to $N_{\mathbf{A}}$.

(c) The induced morphism $\bar{f}: L(M) / q_{\mathrm{s}} L(M) \rightarrow L(N) / q_{\mathrm{s}} L(N)$ sends $B(M)$ to $B(N) \cup\{0\}$.

(d) $\operatorname{Ker}(f)$ is generated by a part of the global basis of $M$. 
In such a case, $f(M)$ has a global basis, and we have

$$
B(M) \supset B(f(M)) \subset B(N) .
$$

If $f$ is a monomorphism then $B(M) \simeq B(f(M)) \subset B(N)$, and if $f$ is an epimorphism then $B(M) \supset B(f(M)) \simeq B(N)$.

\section{§2.6. Extremal vectors}

Let $M$ be an integrable $U_{q}^{-}(\mathfrak{g})$-module. A non-zero vector $u \in M$ of weight $\lambda \in P$ is called extremal (see [13]), if we can find a subset $F$ of non-zero weight vectors in $M$ containing $u$ and satisfying the following properties:

if $v \in F$ and $i$ satisfy $\left\langle h_{i}, \operatorname{wt}(v)\right\rangle \geqslant 0$, then $e_{i} v=0$ and $f_{i}^{\left(\left\langle h_{i}, \operatorname{wt}(v)\right\rangle\right)} v \in F$,

if $v \in F$ and $i$ satisfy $\left\langle h_{i}, \operatorname{wt}(v)\right\rangle \leqslant 0$, then $f_{i} v=0$ and $e_{i}^{\left(-\left\langle h_{i}, \operatorname{wt}(v)\right\rangle\right)} v \in F$,

The Weyl group $W$ acts on the set of extremal vectors by

$$
\begin{aligned}
& \text { if }\left\langle h_{i}, \operatorname{wt}(u)\right\rangle \geqslant 0, \text { then } S_{s_{i}}^{\text {norm }} u=f_{i}^{\left(\left\langle h_{i}, \operatorname{wt}(u)\right)\right.} u, \\
& \text { if }\left\langle h_{i}, \operatorname{wt}(u)\right\rangle \leqslant 0, \text { then } S_{s_{i}}^{\text {norm }} u=e_{i}^{\left(-\left\langle h_{i}, \operatorname{wt}(u)\right\rangle\right)} u .
\end{aligned}
$$

We have $\operatorname{wt}\left(S_{w}^{\text {norm }} u\right)=w \operatorname{wt}(u)$ for $w \in W$. Note that, by $(2.7), S_{w}^{\text {norm }} u$ is equal to $S_{w} u$ up to a non-zero constant multiple.

Similarly, for a vector $b$ of a regular crystal $B$ with weight $\lambda$, we say that $b$ is an extremal vector if it satisfies the following similar conditions:

$$
\begin{aligned}
& \text { if } w \in W \text { and } i \in I \text { satisfy }\left\langle h_{i}, w \lambda\right\rangle \geqslant 0 \text {, then } \tilde{e}_{i} S_{w} b=0 \text {, } \\
& \text { if } w \in W \text { and } i \in I \text { satisfy }\left\langle h_{i}, w \lambda\right\rangle \leqslant 0 \text { then } \tilde{f}_{i} S_{w} b=0 .
\end{aligned}
$$

For $\lambda \in P$, let us denote by $V(\lambda)$ the $U_{q}^{-}(\mathfrak{g})$-module generated by $u_{\lambda}$ with the defining relation that $u_{\lambda}$ is an extremal vector of weight $\lambda$. This is in fact infinitely many linear relations on $u_{\lambda}$.

For a dominant weight $\lambda, V(\lambda)$ is an irreducible highest weight module with highest weight $\lambda$, and $V(-\lambda)$ is an irreducible lowest weight module with lowest weight $-\lambda$.

We proved in $[13]^{1}$ that $V(\lambda)$ has a global basis $(L(\lambda), B(\lambda))$. We denote by the same letter $u_{\lambda}$ the element of $B(\lambda)$ corresponding to $u_{\lambda} \in V(\lambda)$. Moreover $U_{q}^{-}(\mathfrak{g}) a_{\lambda} \rightarrow V(\lambda)\left(a_{\lambda} \mapsto u_{\lambda}\right)$ is compatible with global bases. Hence the crystal

\footnotetext{
${ }^{1} \operatorname{In}[13]$, it is denoted by $V^{\max }(\lambda)$, because I thought there would be a natural $U_{q}^{-}(\mathfrak{g})$ module whose crystal base is the connected component of $B(\lambda)$.
} 
$B(\lambda)$ is isomorphic to the subcrystal of $B(\infty) \otimes t_{\lambda} \otimes B(-\infty)$ consisting of vectors $b$ such that $b^{*}$ is an extremal vector of weight $-\lambda$. By this embedding, $u_{\lambda} \in B(\lambda)$ corresponds to $u_{\infty} \otimes t_{\lambda} \otimes u_{-\infty}$.

Note that

$$
U_{q}^{+}(\mathfrak{g}) u_{\lambda}=\underset{b \in B(\lambda) \cap\left(u_{\infty} \otimes t_{\lambda} \otimes B(-\infty)\right)}{\bigoplus} K G(b) .
$$

For any $w \in W, u_{\lambda} \mapsto S_{w^{-1}}^{\text {norm }} u_{w \lambda}$ gives an isomorphism of $U_{q}^{-}(\mathfrak{g})$-modules:

$$
V(\lambda) \stackrel{\sim}{\longrightarrow} V(w \lambda) .
$$

This is compatible with global bases. Similarly, letting $S_{w}^{*}$ be the Weyl group action on $B\left(\widetilde{U}_{q}(\mathfrak{g})\right)$ with respect to the star crystal structure and regarding $B(\lambda)$ as a subcrystal of $B\left(\widetilde{U}_{q}(\mathfrak{g})\right), S_{w}^{*}: B\left(\widetilde{U}_{q}(\mathfrak{g})\right) \stackrel{\sim}{\longrightarrow} B\left(\widetilde{U}_{q}(\mathfrak{g})\right)$ induces an isomorphism of crystals

$$
S_{w}^{*}: B(\lambda) \stackrel{\sim}{\longrightarrow} B(w \lambda)
$$

This coincides with the crystal isomorphism induced by $V(\lambda) \stackrel{\sim}{\longrightarrow} V(w \lambda)$. Note that we have

$$
S_{w} S_{w}^{*}\left(u_{\infty} \otimes t_{\lambda} \otimes u_{-\infty}\right)=u_{\infty} \otimes t_{w \lambda} \otimes u_{-\infty}
$$

\section{§2.7. Global bases of tensor products}

Let us recall the following results proved by Lusztig ([19]). Let $\mathscr{O}_{\text {int }}$ be the category of integrable $U_{q}^{-}(\mathfrak{g})$-modules which are a direct sum of $V(\lambda)$ 's $\left(\lambda \in P^{+}\right)$. Similarly let $\mathscr{O}_{\text {int }}^{-}$be the category of integrable $U_{q}^{-}(\mathfrak{g})$-modules which are a direct sum of $V(\lambda)^{\text {'s }}\left(\lambda \in P^{-}\right)$. Let $M$ and $N$ be $U_{q}^{-}(\mathfrak{g})$-modules. Assume that $M$ and $N$ have bar involutions, and that either $M \in \mathscr{O}_{\text {int }}$ or $N \in \mathscr{O}_{\text {int }}^{-}$. Then there exists a unique bar involution on $M \otimes N$ such that

$(u \otimes v)^{-}=\bar{u} \otimes \bar{v}$ for every $u \in M$ and $v \in N$ such that either $u$ is a highest weight vector or $v$ is a lowest weight vector.

Assume further that $M$ and $N$ have a global basis. Then $M \otimes N$ has a crystal base $(L(M \otimes N), B(M \otimes N)):=\left(L(M) \otimes_{\mathbf{A}_{0}} L(N), B(M) \otimes B(N)\right)$, and an A-form $(M \otimes N)_{\mathbf{A}}=M_{\mathbf{A}} \otimes_{\mathbf{A}} N_{\mathbf{A}}$. Then $M \otimes N$ has a global basis; namely $\left(L(M \otimes N), L(M \otimes N)^{-},(M \otimes N) \mathbf{A}\right)$ is balanced. In particular, $V(\lambda) \otimes V(\mu)$ has a global basis either if $\lambda$ is dominant or if $-\mu$ is dominant. 
Let $\lambda \in P$. Then for any pair of dominant integral weights $\xi$ and $\eta$ such that $\lambda=\xi-\eta, U_{q}^{-}(\mathfrak{g}) a_{\lambda} \rightarrow V(\xi) \otimes V(-\eta)\left(a_{\lambda} \mapsto u_{\xi} \otimes u_{-\eta}\right)$ is compatible with global bases. Conversely the global basis of $U_{q}^{-}(\mathfrak{g}) a_{\lambda}$ is characterized by the above property.

Lemma 2.5. For $\lambda \in P^{+}$and $\mu \in P$,

$$
U_{q}^{-}(\mathfrak{g}) a_{\lambda+\mu} \rightarrow V(\lambda) \otimes U_{q}^{-}(\mathfrak{g}) a_{\mu} \quad\left(a_{\lambda+\mu} \mapsto u_{\lambda} \otimes a_{\mu}\right)
$$

is compatible with global bases.

Proof. For dominant integral weights $\xi$ and $\eta$ such that $\mu=\xi-\eta$, we have a diagram of morphisms compatible with crystal basses except the dotted arrow:

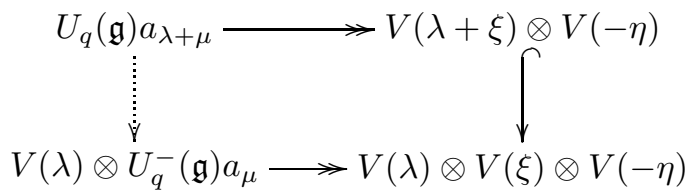

Hence the dotted arrow is compatible with crystal bases.

This morphism (2.27) induces an embedding of crystals

$$
B\left(U_{q}^{-}(\mathfrak{g}) a_{\lambda+\mu}\right) \hookrightarrow B(\lambda) \otimes B\left(U_{q}^{-}(\mathfrak{g}) a_{\mu}\right) \text { for } \lambda \in P^{+} \text {and } \mu \in P .
$$

There exists an embedding $B(\infty) \hookrightarrow B(\lambda) \otimes B(\infty) \otimes T_{-\lambda}$, and the above morphism coincides with the composition

$$
\begin{aligned}
B\left(U_{q}^{-}(\mathfrak{g}) a_{\lambda+\mu}\right) \simeq & B(\infty) \otimes T_{\lambda+\mu} \otimes B(-\infty) \hookrightarrow B(\lambda) \otimes B(\infty) \\
& \otimes T_{-\lambda} \otimes T_{\lambda+\mu} \otimes B(-\infty) \\
\simeq & B(\lambda) \otimes B(\infty) \otimes T_{\mu} \otimes B(-\infty) \simeq B(\lambda) \otimes B\left(U_{q}^{-}(\mathfrak{g}) a_{\mu}\right) .
\end{aligned}
$$

\section{$\S 2.8$. Demazure modules}

Let $M$ be an integrable $U_{q}^{-}(\mathfrak{g})$-module with a global basis $(L(M), B(M)$, $\left.M_{\mathbf{A}},-\right)$. Let $N$ be a $U_{q}^{+}(\mathfrak{g})$-submodule of $M$. We say that $N$ is compatible with the global basis of $M$ if there exists a subset $B(N)$ of $B(M)$ such that $N=\oplus_{b \in B(N)} K G(b)$.

It is shown in [12] that

$$
\begin{aligned}
& \tilde{e}_{i} B(N) \subset B(N) \cup\{0\}, \text { and } U_{q}^{-}(\mathfrak{g}) N=U_{q}^{-}(\mathfrak{g}) N \text { is also } \\
& \text { compatible with the global basis. }
\end{aligned}
$$


Namely there exists a subset $B\left(U_{q}^{-}(\mathfrak{g}) N\right)$ of $B(M)$ such that

$$
U_{q}^{-}(\mathfrak{g}) N=\bigoplus_{b \in B\left(U_{q}^{-}(\mathfrak{g}) N\right)} K G(b) .
$$

Moreover we have

$$
B\left(U_{q}^{-}(\mathfrak{g}) N\right)=\left\{\tilde{f}_{i_{1}} \cdots \tilde{f}_{i_{m}} b ; m \geqslant 0, i_{1}, \ldots i_{m} \in I, b \in B(N)\right\} \backslash\{0\} .
$$

For $\lambda \in P$, the $U_{q}^{ \pm}(\mathfrak{g})$-submodule $U_{q}^{ \pm}(\mathfrak{g}) u_{\lambda}$ of $V(\lambda)$ is compatible with the global basis of $V(\lambda)$ (see (2.25)).

We set

$$
B^{ \pm}(\lambda)=B\left(U_{q}^{ \pm}(\mathfrak{g}) u_{\lambda}\right)
$$

Regarding $B(\lambda)$ as a subset of $B\left(U_{q}^{-}(\mathfrak{g}) a_{\lambda}\right)=B(\infty) \otimes t_{\lambda} \otimes B(-\infty)$, we have $B^{+}(\lambda)=B(\lambda) \cap\left(u_{\infty} \otimes t_{\lambda} \otimes B(-\infty)\right) \quad$ and $\quad B^{-}(\lambda)=B(\lambda) \cap\left(B(\infty) \otimes t_{\lambda} \otimes u_{-\infty}\right)$.

The subset $B^{+}(\lambda)$ satisfies the following properties.

\section{Lemma 2.6.}

(i) $\tilde{e}_{i} B^{+}(\lambda) \subset B^{+}(\lambda) \cup\{0\}$.

(ii) For any $b \in B^{+}(\lambda)$, if $\varepsilon_{i}(b)>0$, then $\tilde{f}_{i} b \in B^{+}(\lambda) \cup\{0\}$. Or equivalently, for any $i$-string $S$ of $B(\lambda), S \cap B^{+}(\lambda)$ is either $S$ itself, the empty set or the set consisting of the highest weight vector of $S$. Here an $i$-string is a connected component with respect to the crystal structure over $U_{q}^{-}(\mathfrak{g})_{i}$.

This is a consequence of the following lemma. Note that $B\left(U_{q}^{+}(\mathfrak{g}) a_{\lambda}\right)=$ $u_{\infty} \otimes T_{\lambda} \otimes B(-\infty)$.

\section{Lemma 2.7.}

(i) $\tilde{e}_{i} B\left(U_{q}^{+}(\mathfrak{g}) a_{\lambda}\right) \subset B\left(U_{q}^{+}(\mathfrak{g}) a_{\lambda}\right) \cup\{0\}$.

(ii) For any $b \in B\left(U_{q}^{+}(\mathfrak{g}) a_{\lambda}\right)$, if $\varepsilon_{i}(b)>0$, then $\tilde{f}_{i} b \in B\left(U_{q}^{+}(\mathfrak{g}) a_{\lambda}\right) \cup\{0\}$. Or equivalently, for any $i$-string $S$ of $B\left(U_{q}^{-}(\mathfrak{g}) a_{\lambda}\right), S \cap B\left(U_{q}^{+}(\mathfrak{g}) a_{\lambda}\right)$ is either $S$ itself, the empty set or the set consisting of the highest weight vector of $S$.

Proof. The first property is evident. In order to prove (ii), write $b=$ $u_{\infty} \otimes t_{\lambda} \otimes b^{\prime}$ with $b^{\prime} \in B(-\infty)$. Then $\varepsilon_{i}(b)=\max \left(0, \varepsilon_{i}\left(t_{\lambda} \otimes b^{\prime}\right)\right)$, and hence $0=\varphi_{i}\left(u_{\infty}\right)<\varepsilon_{i}\left(t_{\lambda} \otimes b^{\prime}\right)$. We have therefore $\tilde{f}_{i} b=u_{\infty} \otimes t_{\lambda} \otimes \tilde{f}_{i} b^{\prime}$.

Similar results hold for $B^{-}(\lambda)$ and $B\left(U_{q}^{-}(\mathfrak{g}) a_{\lambda}\right)$. 
Proposition 2.8. For $\beta \in \Delta_{+}^{\mathrm{re}}$ and $\lambda \in P$, assume $(\beta, \lambda) \geqslant 0$. Then we have

$$
S_{s_{\beta}} u_{\lambda} \in U_{q}^{-}(\mathfrak{g}) u_{\lambda} \quad \text { and } \quad S_{s_{\beta}} u_{\lambda} \in B^{-}(\lambda)
$$

Proof. We shall argue by the induction of ht $(\beta)$. Let us take $i \in I$ such that $\left\langle h_{i}, \beta\right\rangle>0$. If $\beta=\alpha_{i}$ then the assertion is trivial. Otherwise we have $\gamma:=s_{i}(\beta) \in \Delta_{+}^{\mathrm{re}}$. Since $\left(\gamma, s_{i} \lambda\right)=(\beta, \lambda) \geqslant 0$, the induction hypothesis implies that

$$
S_{s_{\gamma}} S_{i} u_{\lambda} \in U_{q}^{-}(\mathfrak{g}) S_{i} u_{\lambda}
$$

If $\left\langle h_{i}, \lambda\right\rangle \geqslant 0$, we have

$$
U_{q}^{-}(\mathfrak{g}) u_{\lambda} \supset U_{q}^{-}(\mathfrak{g}) S_{i} u_{\lambda} \supset U_{q}^{-}(\mathfrak{g}) S_{s_{\gamma}} S_{i} u_{\lambda}=S_{i} S_{s_{\beta}} u_{\lambda}
$$

Since $U_{q}^{-}(\mathfrak{g}) u_{\lambda}$ is an $U_{q}(\mathfrak{g})_{i}$-module, it contains $S_{s_{\beta}} u_{\lambda}$.

Now assume that $\left\langle h_{i}, \lambda\right\rangle<0$. Then $\left\langle h_{i}, s_{\beta} \lambda\right\rangle=\left\langle h_{i}, \lambda\right\rangle-\left\langle\beta^{\vee}, \lambda\right\rangle\left\langle h_{i}, \beta\right\rangle<0$. By (2.29), we have

$$
\begin{aligned}
S_{i} S_{s_{\beta}} S_{i}\left(u_{\infty} \otimes t_{s_{i} \lambda} \otimes u_{-\infty}\right) & =S_{s_{\gamma}}\left(u_{\infty} \otimes t_{s_{i} \lambda} \otimes u_{-\infty}\right) \\
& \in B(\infty) \otimes t_{s_{i} \lambda} \otimes u_{-\infty} .
\end{aligned}
$$

Applying $\tilde{e}_{i}^{* \max }$, we have (here $\tilde{e}_{i}^{* \max } b=\tilde{e}_{i}^{*} \varepsilon_{i}^{*}(b) b$ and $\left.\tilde{f}_{i}^{\max } b=\tilde{f}_{i}^{\varphi_{i}(b)} b\right)$

$$
\begin{aligned}
S_{i} S_{s_{\beta}}\left(u_{\infty} \otimes t_{\lambda} \otimes u_{-\infty}\right) & =\tilde{e}_{i}^{* \max } S_{i} S_{s_{\beta}} S_{i}\left(u_{\infty} \otimes t_{s_{i} \lambda} \otimes u_{-\infty}\right) \\
& \in \tilde{e}_{i}^{* \max }\left(B(\infty) \otimes t_{s_{i} \lambda} \otimes u_{-\infty}\right)
\end{aligned}
$$

and hence

$$
\begin{aligned}
S_{s_{\beta}}\left(u_{\infty} \otimes t_{\lambda} \otimes u_{-\infty}\right) & =\tilde{f}_{i}^{\max } S_{i} S_{s_{\beta}}\left(u_{\infty} \otimes t_{\lambda} \otimes u_{-\infty}\right) \\
& \in \tilde{f}_{i}^{\max } \tilde{e}_{i}^{* \max }\left(B(\infty) \otimes t_{s_{i} \lambda} \otimes u_{-\infty}\right) \\
& \subset \tilde{f}_{i}^{\max }\left(\bigcup_{n \geqslant 0} B(\infty) \otimes t_{\lambda} \otimes \tilde{e}_{i}^{n} u_{-\infty}\right) \\
& \subset B(\infty) \otimes t_{\lambda} \otimes u_{-\infty} .
\end{aligned}
$$

The last inclusion follows from

$$
\tilde{f}_{i}^{\max }\left(b_{1} \otimes t_{\lambda} \otimes b_{2}\right)=b_{1}^{\prime} \otimes t_{\lambda} \otimes \tilde{f}_{i}^{\max } b_{2} \quad \text { for some } b_{1}^{\prime} \in B(\infty) .
$$




\section{§2.9. Affine case}

Until now, we have assumed that $\mathfrak{g}$ is a symmetrizable Kac-Moody algebra. From now on, we assume further that $U_{q}^{-}(\mathfrak{g})$ is a quantized affine algebra.

2.9.1. Extended Weyl groups We take a weight lattice $P$ of $\operatorname{rank} \operatorname{rk}(\mathfrak{g})+1$ and an inner product on $P$ as in [14]. We set $\mathfrak{t}^{*}=\mathbb{Q} \otimes P$, which is canonically determined by the Dynkin diagram.

Let us define $\delta \in \sum_{i} \mathbb{Z}_{\geqslant 0} \alpha_{i}$ and $c \in \sum_{i} \mathbb{Z}_{\geqslant 0} h_{i}$ by

$$
\begin{aligned}
& \left\{\lambda \in \sum_{i} \mathbb{Z} \alpha_{i} ;\left\langle h_{i}, \lambda\right\rangle=0 \text { for every } i \in I\right\}=\mathbb{Z} \delta, \\
& \left\{h \in \sum_{i} \mathbb{Z} h_{i} ;\left\langle h, \alpha_{i}\right\rangle=0 \text { for every } i \in I\right\}=\mathbb{Z} c .
\end{aligned}
$$

By the inner product of $\mathfrak{t}^{*}$, we sometimes identify $\mathfrak{t}^{*}$ and its dual. Note that the inner product on $\mathfrak{t}^{*}$ is so normalized that $\delta$ and $c$ correspond by this identification.

For $\alpha \in \Delta^{\text {re }}$, we set $c_{\alpha}:=\max (1,(\alpha, \alpha) / 2) \in \mathbb{Z}$. Then we have

$$
(\alpha+\mathbb{Z} \delta) \cap \Delta=\alpha+c_{\alpha} \mathbb{Z} .
$$

Let us denote by $P_{\mathrm{cl}}$ the quotient space $P /(P \cap \mathbb{Q} \delta)$, and let us denote by $\mathrm{cl}: P \rightarrow P_{\mathrm{cl}}$ the canonical projection. Let us denote by $P_{\mathrm{cl}}^{*}$ the dual lattice of $P_{\mathrm{cl}}$, i.e. $P_{\mathrm{cl}}^{*}=\operatorname{Ker}\left(\delta: P^{*} \rightarrow \mathbb{Z}\right)=\left(\sum_{i} \mathbb{Q} h_{i}\right) \cap P^{*}$.

Similarly to $P_{\mathrm{cl}}$, we define $\mathfrak{t}_{\mathrm{cl}}^{*}:=\mathfrak{t}^{*} / \mathbb{Q} \delta$, and let cl: $\mathfrak{t}^{*} \rightarrow \mathfrak{t}_{\mathrm{cl}}^{*}$ be the canonical projection. Define $\mathfrak{t}^{* 0}:=\operatorname{Ker}\left(c: \mathfrak{t}^{*} \rightarrow \mathbb{Q}\right)$, and $\mathfrak{t}_{\mathrm{cl}}^{* 0}=\operatorname{cl}\left(\mathfrak{t}^{* 0}\right)$. The dimension of $\mathfrak{t}_{\mathrm{cl}}^{* 0}$ is equal to $\mathrm{rk}(\mathfrak{g})-1$. The inner product of $\mathfrak{t}^{*}$ induces a positive definite inner product on $\mathfrak{t}_{\mathrm{cl}}^{* 0}$.

Let us denote by $\mathrm{O}\left(\mathfrak{t}^{*}\right)$ the orthogonal group, and $\mathrm{O}\left(\mathfrak{t}^{*}\right)_{\delta}:=$ $\left\{g \in \mathrm{O}\left(\mathfrak{t}^{*}\right) ; g \delta=\delta\right\}$ the isotropy subgroup at $\delta$. Then there is an exact sequence

$$
1 \longrightarrow \mathfrak{t}_{\mathrm{cl}}^{* 0} \stackrel{t}{\longrightarrow} \mathrm{O}\left(\mathfrak{t}^{*}\right)_{\delta} \stackrel{\mathrm{cl}_{0}}{\longrightarrow} \mathrm{O}\left(\mathfrak{t}_{\mathrm{cl}}^{* 0}\right) \longrightarrow 1 .
$$

Here $t: \mathfrak{t}_{\mathrm{cl}}{ }^{0} \rightarrow \mathrm{O}\left(\mathfrak{t}^{*}\right)_{\delta}$ is given by

$$
t(\operatorname{cl}(\xi))(\lambda)=\lambda+(\lambda, \delta) \xi-(\lambda, \xi) \delta-\frac{(\xi, \xi)}{2}(\lambda, \delta) \delta \quad \text { for } \xi \in \mathfrak{t}^{* 0} \text { and } \lambda \in \mathfrak{t}^{*} .
$$

Let us set $W_{\mathrm{cl}}=\mathrm{cl}_{0}(W)$. Then $W_{\mathrm{cl}}$ is the Weyl group of the root system $\Delta_{\mathrm{cl}}:=\operatorname{cl}\left(\Delta^{\mathrm{re}}\right) \subset \mathfrak{t}_{\mathrm{cl}}^{* 0}$. We define the extended Weyl group $\widetilde{W}$ by

$$
\widetilde{W}:=\left\{w \in \mathrm{O}\left(\mathrm{t}^{*}\right)_{\delta} ; w \Delta=\Delta \text { and } \operatorname{cl}_{0}(w) \in W_{\mathrm{cl}}\right\} .
$$


Then we have a commutative diagram with the exact rows:

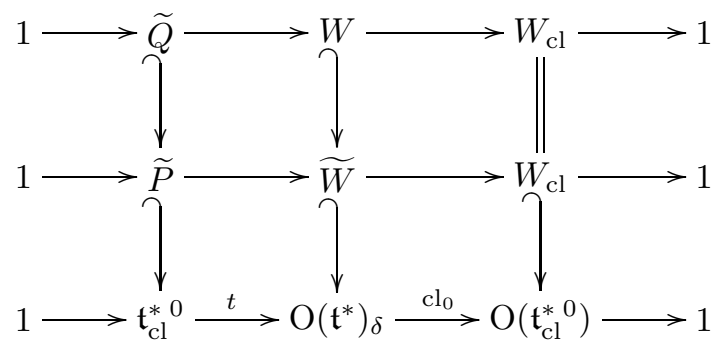

Here $\widetilde{P}$ and $\widetilde{Q}$ are given by

$$
\widetilde{P}=P_{\mathrm{cl}}^{0} \cap P_{\mathrm{cl}}^{\vee 0} \quad \text { and } \quad \widetilde{Q}=Q_{\mathrm{cl}} \cap Q_{\mathrm{cl}}^{\vee},
$$

where

$$
\begin{aligned}
P_{\mathrm{cl}}^{0} & :=\left\{\lambda \in \mathfrak{t}_{\mathrm{cl}}^{* 0} ;\left\langle h_{i}, \lambda\right\rangle \in \mathbb{Z} \text { for every } i \in I\right\}, \\
P_{\mathrm{cl}}^{0 \vee} & :=\left\{\lambda \in \mathfrak{t}_{\mathrm{cl}}^{* 0} ;\left(\alpha_{i}, \lambda\right) \in \mathbb{Z} \text { for every } i \in I\right\}, \\
Q_{\mathrm{cl}} & :=\sum_{i \in I} \mathbb{Z} \operatorname{cl}\left(\alpha_{i}\right), \\
Q_{\mathrm{cl}}^{\vee} & :=\sum_{i \in I} \mathbb{Z} \operatorname{cl}\left(h_{i}\right) .
\end{aligned}
$$

The Weyl group $W$ is a normal subgroup of $\widetilde{W}$, and $\widetilde{W}$ is a semi-direct product of $W$ and $\operatorname{Aut}_{0}(\mathrm{Dyn}):=\{\iota ; \iota$ is a Dynkin diagram automorphism such that $\}$ $\operatorname{cl}_{0}(\iota) \in W_{\mathrm{cl}}$.

$$
\widetilde{P} / \widetilde{Q} \stackrel{\sim}{\longrightarrow} \widetilde{W} / W \stackrel{\sim}{\longrightarrow} \operatorname{Aut}_{0}(\mathrm{Dyn}) .
$$

Remark 2.9 .

(i) If $\mathfrak{g}$ is untwisted, then $(\alpha, \alpha) / 2 \leqslant 1$ for every $\alpha \in \Delta^{\text {re }}$ and

$$
\widetilde{P}=P_{\mathrm{cl}}^{0 \vee} \subset P_{\mathrm{cl}}^{0}, \quad \widetilde{Q}=Q_{\mathrm{cl}}^{\vee} \subset Q_{\mathrm{cl}} .
$$

(ii) If $\mathfrak{g}$ is the dual of an untwisted affine algebra, then $(\alpha, \alpha) / 2 \geqslant 1$ for every $\alpha \in \Delta^{\text {re }}$ and

$$
\widetilde{P}=P_{\mathrm{cl}}^{0} \subset P_{\mathrm{cl}}^{0 \vee}, \quad \widetilde{Q}=Q_{\mathrm{cl}} \subset Q_{\mathrm{cl}}^{\vee}
$$

(iii) If $\mathfrak{g}=A_{2 n}^{(2)}$, then we have $(\alpha, \alpha) / 2=1 / 2,1$ or 2 , and

$$
\widetilde{P}=\widetilde{Q}=P_{\mathrm{cl}}^{0}=P_{\mathrm{cl}}^{0 \vee}=Q_{\mathrm{cl}}=Q_{\mathrm{cl}}^{\vee}=\sum_{\alpha \in \Delta^{\mathrm{re}}} \mathbb{Z} \operatorname{cl}(\alpha)=\sum_{\alpha \in \Delta^{\mathrm{re}},(\alpha, \alpha) / 2=1} \mathbb{Z} \operatorname{cl}(\alpha) .
$$


2.9.2. Peter-Weyl theorem Let us recall some of the results by Nakajima and Beck-Nakajima.

The following theorem is conjectured in [14] by the author and proved in [2] by Beck-Nakajima.

Theorem 2.10 (a version of Peter-Weyl theorem).

$$
B\left(\widetilde{U}_{q}(\mathfrak{g})\right) \simeq\left(\bigsqcup_{\lambda \in P} B(\lambda) \times B_{0}(-\lambda)\right) / W .
$$

Here $B_{0}(\lambda)$ is the connected component of $B(\lambda)$ containing $u_{\lambda}$. Note that $B_{0}(\lambda)=B(\lambda)$ when the level of $\lambda$ does not vanish. The Weyl group acts on $\bigsqcup_{\lambda \in P} B(\lambda) \times B_{0}(-\lambda)$ by $W \ni w: B(\lambda) \times B_{0}(-\lambda) \rightarrow B(w \lambda) \times B_{0}(-w \lambda)$ via the action given in (2.26). The left crystal structure $\left(\tilde{e}_{i}, \tilde{f}_{i}\right)$ on $B\left(\widetilde{U}_{q}(\mathfrak{g})\right)$ is compatible with the crystal structure of $B(\lambda)$, the first factor of $B(\lambda) \times B_{0}(-\lambda)$, and the right crystal structure $\left(\tilde{e}_{i}^{*}, \tilde{f}_{i}^{*}\right)$ on $B\left(\widetilde{U}_{q}(\mathfrak{g})\right)$ is compatible with the crystal structure of $B_{0}(-\lambda)$, the second factor of $B(\lambda) \times B_{0}(-\lambda)$.

For $\lambda \in P$, there exists a unique symmetric bilinear form $(\cdot, \cdot)$ on $V(\lambda)$ that satisfies:

$$
\begin{aligned}
\left(u_{\lambda}, G(b)\right) & =\delta_{b, u_{\lambda}} \text { for every } b \in B(\lambda) \\
\left(e_{i} u, v\right) & =\left(u, f_{i} v\right) \text { for every } u, v \in V(\lambda), \\
(q(h) u, v) & =(u, q(h) v) \text { for every } u, v \in V(\lambda) \text { and } h \in d^{-1} P^{*} .
\end{aligned}
$$

The following theorem is trivial for non-zero level case, and proved in [21, 2] by Nakajima and Beck-Nakajima for the zero level case.

\section{Theorem 2.11.}

(i) This symmetric bilinear form on $V(\lambda)$ is non-degenerate.

(ii) $\left(G(b), G\left(b^{\prime}\right)\right) \in q^{(\mu, \mu)-(\lambda, \lambda)}\left(\delta_{b, b^{\prime}}+q_{\mathrm{s}} \mathbf{A}_{0}\right)$ for any $\mu \in P$ and $b, b^{\prime} \in B(\lambda)_{\mu}$.

(iii) For $b, b^{\prime} \in B(\lambda)_{\lambda}$, we have $\left(G(b), G\left(b^{\prime}\right)\right)=\delta_{b, b^{\prime}}$.

In particular if $v$ is a non-zero vector of $V(\lambda)$, then there exists $P \in U_{q}^{-}(\mathfrak{g})$ such that $\left(u_{\lambda}, P v\right)$ does not vanish. Note that $\left(u_{\lambda}, P v\right)$ coincides with the coefficient of $u_{\lambda}$ when we write $P v$ as a linear combination of the global basis.

Conjecture 2.12. Theorem 2.11 holds for an arbitrary symmetrizable Kac-Moody Lie algebra $\mathfrak{g}$. 


\section{§3. Extremal Vectors}

We assume that $U_{q}^{-}(\mathfrak{g})$ is a quantized affine algebra. Let $M$ be an integrable $U_{q}^{-}(\mathfrak{g})$-module with a global basis. Let $N$ be a $U_{q}^{+}(\mathfrak{g})$-submodule of $M$ compatible with the global basis of $M$. Then, for $\lambda \in P^{+}, u_{\lambda} \otimes N$ is also a $U_{q}^{+}(\mathfrak{g})$-submodule of $V(\lambda) \otimes M$ compatible with the global basis. Hence $U_{q}^{-}(\mathfrak{g})\left(u_{\lambda} \otimes N\right)$ is a $U_{q}^{-}(\mathfrak{g})$-module compatible with the global basis of $V(\lambda) \otimes M$.

Proposition 3.1. Assume that for any $b \in B(N)$ if $\varepsilon_{i}(b)>0$, then $\tilde{f}_{i} b \in B(N) \cup\{0\}$. Or equivalently, for any $i$-string $S$ of $B(M), S \cap B(N)$ is either $S$ itself, the empty set or the set consisting of the highest weight vector of $S$. Assume further that $f_{i} N \subset N$ whenever $\left\langle h_{i}, \lambda\right\rangle=0$. Then we have

$$
U_{q}^{-}(\mathfrak{g})\left(u_{\lambda} \otimes N\right) \cap u_{\lambda} \otimes M=u_{\lambda} \otimes N .
$$

Proof. It is enough to show that

$$
B\left(U_{q}^{-}(\mathfrak{g})\left(u_{\lambda} \otimes N\right)\right) \cap u_{\lambda} \otimes B(M)=u_{\lambda} \otimes B(N) .
$$

We have

$$
B\left(U_{q}^{-}(\mathfrak{g})\left(u_{\lambda} \otimes N\right)\right)=\left\{\tilde{f}_{i_{1}} \cdots \tilde{f}_{i_{m}} b ; m \geqslant 0, i_{1}, \ldots i_{m} \in I, b \in u_{\lambda} \otimes B(N)\right\} \backslash\{0\} .
$$

Hence it is enough to show that, for $b \in B(N)$ such that $\tilde{f}_{i}\left(u_{\lambda} \otimes b\right)=u_{\lambda} \otimes \tilde{f}_{i} b$, we have $\tilde{f}_{i} b \in B(N) \cup\{0\}$. Since $\tilde{f}_{i}\left(u_{\lambda} \otimes b\right)=u_{\lambda} \otimes \tilde{f}_{i} b$ if and only if $\left\langle h_{i}, \lambda\right\rangle \leqslant \varepsilon_{i}(b)$, and $\tilde{f}_{i} B(N) \subset B(N) \cup\{0\}$ if $\left\langle h_{i}, \lambda\right\rangle=0$, the assertion follows.

Corollary 3.2. For $\lambda \in P^{+}$and $\mu \in P$ such that $\left\langle h_{i}, \mu\right\rangle \leqslant 0$ whenever $\left\langle h_{i}, \lambda\right\rangle=0$, we have

$$
U_{q}^{-}(\mathfrak{g})\left(u_{\lambda} \otimes u_{\mu}\right) \cap u_{\lambda} \otimes V(\mu)=u_{\lambda} \otimes U_{q}^{+}(\mathfrak{g}) u_{\mu},
$$

and

$$
B\left(U_{q}^{-}(\mathfrak{g})\left(u_{\lambda} \otimes u_{\mu}\right)\right) \cap u_{\lambda} \otimes B(\mu)=u_{\lambda} \otimes B^{+}(\mu) .
$$

Indeed, Lemma 2.6 allows us to apply the proposition above to $M=V(\mu)$ and $N=U_{q}^{+}(\mathfrak{g}) u_{\mu}$.

Let $\lambda \in P^{+}$be a dominant integral weight and $\mu \in P$ an integral weight. Then we have a chain of morphisms compatible with global bases

$$
U_{q}^{-}(\mathfrak{g}) a_{\lambda+\mu} \rightarrow V(\lambda) \otimes U_{q}^{-}(\mathfrak{g}) a_{\mu} \rightarrow V(\lambda) \otimes V(\mu)
$$


Theorem 3.3. Let $\lambda \in P^{+}$be a dominant integral weight and $\mu \in P$ an integral weight. Then there exist a unique homomorphism $U_{q}^{-}(\mathfrak{g})\left(u_{\lambda} \otimes u_{\mu}\right) \rightarrow$ $V(\lambda+\mu)$ that sends $u_{\lambda} \otimes u_{\mu}$ to $u_{\lambda+\mu}$. Moreover this morphism is compatible with global bases.

Proof. We have a commutative diagram

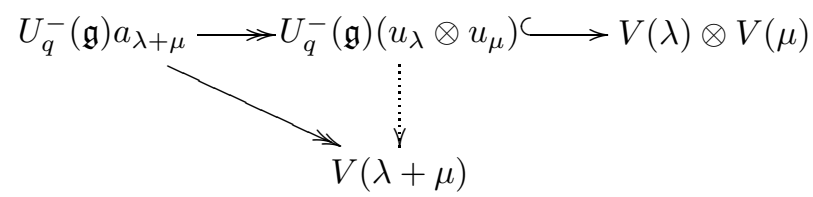

All the solid arrows are compatible with global bases. Hence, in order to show the theorem, it is enough to show the existence of the dotted arrow.

Correspondingly, we obtain the following diagram of crystal bases.

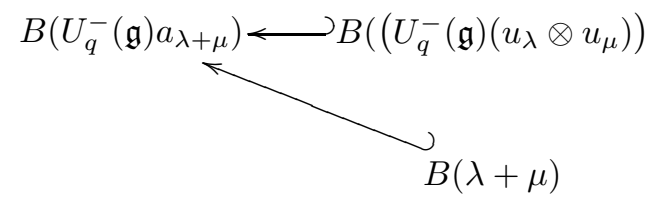

Let $G(b) \in U_{q}^{-}(\mathfrak{g}) a_{\lambda+\mu}$ be the global basis vector corresponding to $b \in B\left(U_{q}^{-}(\mathfrak{g})\right.$ $a_{\lambda+\mu}$ ). Then $b \in B(\lambda+\mu)$ means that $G(b) u_{\lambda+\mu} \neq 0$ (we regard $V(\lambda+\mu)$ as an $\widetilde{U}_{q}(\mathfrak{g})$-module), and $b \in B\left(U_{q}^{-}(\mathfrak{g})\left(u_{\lambda} \otimes u_{\mu}\right)\right)$ means $G(b)\left(u_{\lambda} \otimes u_{\mu}\right) \neq 0$.

Hence we have reduced the problem to the following proposition:

$$
B(\lambda+\mu) \subset B\left(U_{q}^{-}(\mathfrak{g})\left(u_{\lambda} \otimes u_{\mu}\right)\right) \text { as subsets of } B\left(U_{q}^{-}(\mathfrak{g}) a_{\lambda+\mu}\right) .
$$

Taking $b \in B(\lambda+\mu) \subset B\left(U_{q}^{-}(\mathfrak{g}) a_{\lambda+\mu}\right)$, let us show that $b \in B\left(U_{q}^{-}(\mathfrak{g})\left(u_{\lambda} \otimes u_{\mu}\right)\right)$.

Since $G(b) u_{\lambda+\mu} \neq 0$, Theorem 2.11 implies that there exists $P \in U_{q}^{-}(\mathfrak{g})$ such that, when we write $P G(b) u_{\lambda+\mu}$ as a linear combinations of the global basis of $V(\lambda+\mu)$, the coefficient of $u_{\lambda+\mu}$ does not vanish. Hence, if we write $P G(b)$ as a linear combinations of the global basis of $U_{q}^{-}(\mathfrak{g}) a_{\lambda+\mu}$ the coefficient of $a_{\lambda+\mu}$ does not vanish. Hence $P G(b)\left(u_{\lambda} \otimes u_{\mu}\right)$ as a linear combinations of the global basis of $V(\lambda) \otimes V(\mu)$ the coefficient of $u_{\lambda} \otimes u_{\mu}$ does not vanish. We conclude then that $P G(b)\left(u_{\lambda} \otimes u_{\mu}\right) \neq 0$. Hence $b \in B\left(U_{q}^{-}(\mathfrak{g})\left(u_{\lambda} \otimes u_{\mu}\right)\right)$.

Remark 3.4. Theorem 3.3 holds also for any finite-dimensional $\mathfrak{g}$, because Theorem 2.11 trivially holds in such a case.

Corollary 3.5. If $\lambda \in P^{+}$and $\mu \in P$, then $u_{\lambda} \otimes u_{\mu} \in B(\lambda) \otimes B(\mu)$ is an extremal vector, and we have an inclusion $B(\lambda+\mu) \subset B(\lambda) \otimes B(\mu)$ as subsets of $B(\lambda) \otimes B\left(U_{q}^{-}(\mathfrak{g}) a_{\mu}\right)$. 
Note that the first statement holds for an arbitrary Kac-Moody Lie algebra.

\section{§4. Fundamental Representations}

Write the smallest positive imaginary root $\delta$ and the smallest positive imaginary coroot $c$ as

$$
\delta=\sum_{i} a_{i} \alpha_{i}, \quad c=\sum_{i} a_{i}^{\vee} h_{i}
$$

Then we have

$$
a_{i}^{\vee}=\frac{\left(\alpha_{i}, \alpha_{i}\right)}{2} a_{i}
$$

We choose $0 \in I$ such that

(i) Setting $I_{0}=\{i \in I ; i \neq 0\}$ and $W_{0}:=\left\langle s_{i} ; i \in I_{0}\right\rangle \subset W$, the composition $W_{0} \hookrightarrow W \stackrel{\mathrm{cl}_{0}}{\longrightarrow} W_{\mathrm{cl}}$ is an isomorphism.

(ii) $a_{0}=1$.

Such a 0 exists and is unique up to a Dynkin diagram automorphism.

If $\mathfrak{g}=A_{2 n}^{(2)}, \alpha_{0}$ is the longest simple root and $a_{0}^{\vee}=2$.

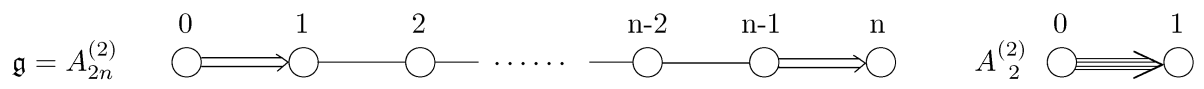

$$
\begin{aligned}
& \delta=\alpha_{0}+2\left(\alpha_{1}+\cdots+\alpha_{n}\right), \\
& c=2\left(h_{0}+\cdots+h_{n-1}\right)+h_{n} . \\
& \left(\alpha_{0}, \alpha_{0}\right)=4,\left(\alpha_{n}, \alpha_{n}\right)=1,\left(\alpha_{i}, \alpha_{i}\right)=2 \text { for } 0<i<n
\end{aligned}
$$

Figure 1. $\mathfrak{g}=A_{2 n}^{(2)}$

If $\mathfrak{g}$ is not of type $A_{2 n}^{(2)}$, then $a_{0}^{\vee}=1$. Note that $\delta-\alpha_{0} \in \Delta^{\text {re }}$ if $\mathfrak{g}$ is not of type $A_{2 n}^{(2)}$, and $\left(\delta-\alpha_{0}\right) / 2 \in \Delta^{\text {re }}$ if $\mathfrak{g}$ is of type $A_{2 n}^{(2)}$. Hence one has always $s_{\delta-\alpha_{0}} \in W$.

Let us denote by $U_{q}\left(\mathfrak{g}_{0}\right)$ the subalgebra of $U_{q}^{-}(\mathfrak{g})$ generated by $e_{i}, f_{i}(i \in$ $\left.I_{0}\right)$. This is the quantized universal enveloping algebra associated with a finitedimensional simple Lie algebra.

Let $\varpi_{k}$ be a fundamental weight of level 0 . That is, $\left\{\varpi_{k}\right\}_{k \in I_{0}}$ is a set of vectors such that $\left\langle h_{j}, \varpi_{k}\right\rangle=0$ for $j \in I_{0}$ with $j \neq k$, and

$$
\begin{aligned}
P_{\mathrm{cl}}^{0+} & :=\left\{\lambda \in \mathfrak{t}_{\mathrm{cl}}^{*} ;\langle c, \lambda\rangle=0 \text { and }\left\langle h_{i}, \lambda\right\rangle \in \mathbb{Z}_{\geqslant 0} \text { for every } i \in I_{0}\right\} \\
& =\sum_{k \in I_{0}} \mathbb{Z}_{\geqslant 0} \varpi_{k} .
\end{aligned}
$$


A fundamental weight of level 0 is unique up to $\mathbb{Q} \delta$. We can take

$$
\varpi_{k}= \begin{cases}\Lambda_{k}-a_{k}^{\vee} \Lambda_{0} & \text { when } a_{0}^{\vee}=1, \\ \frac{2}{\left(\alpha_{k}, \alpha_{k}\right)} \Lambda_{k}-\Lambda_{0} & \text { when } \mathfrak{g}=A_{2 n}^{(2)} .\end{cases}
$$

Here $\Lambda_{k}$ is a vector in $P$ satisfying $\left\langle h_{i}, \Lambda_{k}\right\rangle=\delta_{i k}$ for $i \in I$.

Let $k \in I \backslash\{0\}$. Set $c_{k}=\max \left(1,\left(\alpha_{k}, \alpha_{k}\right) / 2\right) \in \mathbb{Z}$. Then we have

$$
\left\{n \in \mathbb{Z} ; \alpha_{k}+n \delta \in \Delta\right\}=\mathbb{Z} c_{k},
$$

and

$$
W \varpi_{k} \cap\left(\varpi_{k}+\mathbb{Z} \delta\right)=\varpi_{k}+\mathbb{Z} c_{k} \delta .
$$

We have $\varpi_{k}+n \delta \in \mathrm{Wt}\left(V\left(\varpi_{k}\right)\right)$ if and only if $n \in c_{k} \mathbb{Z}$.

For any $\xi=w \lambda \in W \lambda$, we use the notation $u_{\xi}$ for the extremal vector $S_{w}^{\text {norm }} u_{\lambda} \in V(\lambda)$. Note that $S_{w}^{\text {norm }} u_{\lambda}$ is a unique global basis vector of weight $\xi$.

We denote by $U_{q}^{\prime}(\mathfrak{g})$ the subalgebra of $U_{q}^{-}(\mathfrak{g})$ generated by $e_{i}, f_{i}(i \in I)$ and $q(h)\left(h \in d^{-1} P_{\mathrm{cl}}^{*} \subset d^{-1} P^{*}\right)$.

Then there exists a unique $U_{q}^{\prime}(\mathfrak{g})$-morphism $z_{k}: V\left(\varpi_{k}\right) \rightarrow V\left(\varpi_{k}\right)$ sending $u_{\varpi_{k}}$ to $u_{\varpi_{k}+c_{k} \delta}$. The operator $z_{k}$ has weight $c_{k} \delta$. The global basis of $V\left(\varpi_{k}\right)$ is stable by $z_{k}$. We have

$$
z_{k} u_{\xi}=u_{\xi+c_{k} \delta} \quad \text { for every } \xi \in W \cdot \lambda .
$$

The quotient $W\left(\varpi_{k}\right):=V\left(\varpi_{k}\right) /\left(z_{k}-1\right) V\left(\varpi_{k}\right)$ is an irreducible $U_{q}^{\prime}(\mathfrak{g})$ module with a global basis. The morphism $V\left(\varpi_{k}\right) \rightarrow W\left(\varpi_{k}\right)$ sends the members of the global basis of $V\left(\varpi_{k}\right)$ to the one of $W\left(\varpi_{k}\right)$.

In this section, we set

(4.2) $\quad \lambda:=\varpi_{k}, \quad \mu:=w_{0} \varpi_{k} \quad$ where $w_{0}$ be the longest element of $W_{0}$.

Then $\mu \equiv-\varpi_{k^{\prime}} \bmod \mathbb{Z} \delta$ for some $k^{\prime} \in I_{0}$.

Then $U_{q}\left(\mathfrak{g}_{0}\right) u_{\lambda}=U_{q}\left(\mathfrak{g}_{0}\right) u_{\mu}$ is an irreducible $U_{q}\left(\mathfrak{g}_{0}\right)$-module with highest weight $\lambda$ and lowest weight $\mu$. Note that $U_{q}^{+}(\mathfrak{g}) u_{\mu}$ is a $U_{q}\left(\mathfrak{g}_{0}\right)$-module. We have

$$
u_{\xi} \in U_{q}^{+}(\mathfrak{g}) u_{\mu} \text { for any } \xi \in W_{0} \cdot \lambda=W \cdot \lambda \cap\left(\lambda+\sum_{i \in I_{0}} \mathbb{Z} \alpha_{i}\right) .
$$

Lemma 4.1. $z_{k} u_{\mu} \in U_{q}^{+}(\mathfrak{g}) u_{\mu}$.

Proof. Assume $a_{0}^{\vee}=1$. Then we have $\tilde{\alpha}_{k^{\prime}}:=c_{k} \alpha_{k^{\prime}}^{\vee} \in \widetilde{Q}$ and $t\left(\tilde{\alpha}_{k^{\prime}}\right)(\mu)=$ $\mu+c_{k} \delta$. Hence $z_{k} u_{\mu}=S_{t\left(\tilde{\alpha}_{k^{\prime}}\right)}^{\text {norm }} u_{\mu}$. Since we have $c_{k} \delta-\alpha_{k^{\prime}} \in \Delta_{+}^{\mathrm{re}}, t\left(\tilde{\alpha}_{k^{\prime}}\right)=$ 
$s_{c_{k} \delta-\alpha_{k^{\prime}}} s_{\alpha_{k^{\prime}}}$, and $\left(\alpha_{k^{\prime}}, \mu\right)=\left(c_{k} \delta-\alpha_{k^{\prime}}, s_{k^{\prime}} \mu\right)<0$, Proposition 2.8 implies that $S_{t\left(\tilde{\alpha}_{k^{\prime}}\right)}^{\text {norm }} u_{\mu} \in U_{q}^{+}(\mathfrak{g}) u_{\mu}$.

Now assume that $\mathfrak{g}=A_{2 n}^{(2)}$. Then $t\left(-\alpha_{0} / 2\right) \mu=\mu+\delta$ and $t\left(-\alpha_{0} / 2\right)=$ $s_{\alpha_{0}} s_{\left(\delta-\alpha_{0}\right) / 2}$, and $\left(\delta-\alpha_{0}, \mu\right)=\left(\alpha_{0}, s_{\left(\delta-\alpha_{0}\right) / 2} \mu\right)<0$. Note that $\left(\delta-\alpha_{0}\right) / 2 \in \Delta_{+}^{\mathrm{re}}$. Hence Proposition 2.8 implies that $S_{t\left(\tilde{\alpha}_{0}\right)}^{\text {norm }} u_{\mu} \in U_{q}^{+}(\mathfrak{g}) u_{\mu}$.

\section{Lemma 4.2.}

(i) $B^{+}(\mu) \backslash z_{k} B^{+}(\mu) \simeq B(W(\lambda))$ as a crystal over $\mathfrak{g}_{0}$.

(ii) $U_{q}^{+}(\mathfrak{g}) u_{\mu} / U_{q}^{+}(\mathfrak{g}) z_{k} u_{\mu}$ is isomorphic to $W(\lambda)$ as a $U_{q}\left(\mathfrak{g}_{0}\right)$-module

Proof. The crystal $B^{+}(\mu)$ is a regular crystal over $\mathfrak{g}_{0}$. The crystal $B^{+}(\mu)$ is invariant by $z_{k}$, and $\bigcup_{n \in \mathbb{Z}} z_{k}^{n} B^{+}(\mu)=B(\mu), \bigcap_{n \in \mathbb{Z}} z_{k}^{n} B^{+}(\mu)=\emptyset$. On the other hand, the crystal $B(W(\lambda))$ is isomorphic to the quotient of $B(\mu)$ by the action of $\mathbb{Z}$ given by $z_{k}$. Hence $B^{+}(\mu) \backslash z_{k} B^{+}(\mu) \rightarrow B(W(\lambda))$ is bijective. (ii) follows from (i).

Lemma 4.3. Assume that $\left\langle h_{0}, \lambda\right\rangle=-1$ (i.e. $a_{k}^{\vee}=1$ or $\left.\mathfrak{g}=A_{2 n}^{(2)}\right)$.

(i) $c_{k}=1$.

(ii) $W(\lambda)$ is an irreducible $U_{q}\left(\mathfrak{g}_{0}\right)$-module.

Proof. (i) if $\mathfrak{g}=A_{2 n}^{(2)}$, then $\left(\alpha_{k}, \alpha_{k}\right) / 2 \leqslant 1$ for $k \neq 0$, and hence $c_{k}=1$. If $a_{k}^{\vee}=1$, then $1=a_{k}^{\vee}=\frac{\left(\alpha_{k}, \alpha_{k}\right)}{2} a_{k} \geqslant \frac{\left(\alpha_{k}, \alpha_{k}\right)}{2}$, and hence $c_{k}=1$.

(ii) By Lemma 4.2, it is enough to show that $N:=U_{q}^{+}(\mathfrak{g}) u_{\mu} / U_{q}^{+}(\mathfrak{g}) z_{k} u_{\mu}$ is irreducible as a $U_{q}\left(\mathfrak{g}_{0}\right)$-module. Note that $e_{0} u_{\lambda}=u_{s_{o} \lambda}=u_{\lambda+\delta}=z_{k} u_{\lambda} \in$ $U_{q}^{+}(\mathfrak{g}) z_{k} u_{\mu}$ and $e_{i} u_{\lambda}=0$ for $i \neq 0$. Since $U_{q}^{+}(\mathfrak{g}) u_{\mu}=U_{q}^{+}(\mathfrak{g}) U_{q}\left(\mathfrak{g}_{0}\right) u_{\lambda}=$ $U_{q}\left(\mathfrak{g}_{0}\right) U_{q}^{+}(\mathfrak{g}) u_{\lambda}$, we have $N=U_{q}\left(\mathfrak{g}_{0}\right) u_{\lambda} \bmod z_{k} U_{q}^{+}(\mathfrak{g}) u_{\mu}$.

Two vectors $u_{\Lambda_{0}} \otimes u_{\lambda}$ and $u_{\Lambda_{0}} \otimes u_{\mu}$ are extremal vectors in the same connected component of $B\left(\Lambda_{0}\right) \otimes B(\lambda)$, because $u_{\Lambda_{0}}$ belongs to the trivial representation with respect to $\mathfrak{g}_{0}$ and $\mu \in W_{0} \lambda$. Since the level of $\Lambda_{0}+\mu$ is equal to one, there exists a unique dominant weight $\xi_{0}$ of level one such that $\xi_{0} \in W \cdot\left(\Lambda_{0}+\lambda\right)=W \cdot\left(\Lambda_{0}+\mu\right)$. By Corollary 3.5, the connected component of $B\left(\Lambda_{0}\right) \otimes B(\lambda)$ containing $u_{\Lambda_{0}} \otimes u_{\lambda}$ is isomorphic to $B\left(\xi_{0}\right)$. Set $M=V\left(\Lambda_{0}\right) \otimes V(\mu)$ and $M_{n}=U_{q}^{-}(\mathfrak{g})\left(u_{\Lambda_{0}} \otimes z_{k}^{n} u_{\lambda}\right)=U_{q}^{-}(\mathfrak{g})\left(u_{\Lambda_{0}} \otimes z_{k}^{n} u_{\mu}\right) \subset M$. Note that Corollary 3.2 implies

$$
M_{n} \cap\left(u_{\Lambda_{0}} \otimes V(\lambda)\right)=u_{\Lambda_{0}} \otimes U_{q}^{+}(\mathfrak{g}) z_{k}^{n} u_{\mu}
$$


Lemma 4.4.

(i) $M_{n+1} \subset M_{n}$.

(ii) $M=\bigcup_{n \in \mathbb{Z}} M_{n}$.

(iii) $\bigcap_{n \in \mathbb{Z}} M_{n}=0$.

Proof. (i) is obvious. Since $W(\mu)$ is generated by $u_{\mu}$ as an $U_{q}^{+}(\mathfrak{g})$-module (by [1, Proposition 1.16]), we have $V(\mu)=\cup_{n} U_{q}^{+}(\mathfrak{g}) z_{k}^{n} u_{\mu}$, which implies (ii).

In order to prove (iii), it is enough to show that $\cap_{n} B\left(M_{n}\right)=\emptyset$. Any vector $b \in \cap_{n} B\left(M_{n}\right)$ is connected with a vector in $u_{\Lambda_{0}} \otimes B(\mu)$. Since $B\left(M_{n}\right) \cap\left(u_{\Lambda_{0}} \otimes\right.$ $B(\mu))=u_{\Lambda_{0}} \otimes z_{k}^{n} B^{+}(\mu)$, the result follows from $\cap_{n} z_{k}^{n} B^{+}(\mu)=\emptyset$, which is an immediate consequence of $\cap_{n}\left(\mu+n \delta+Q_{+}\right)=\emptyset$.

Lemma 4.5. $B\left(M_{0}\right) \backslash B\left(M_{1}\right) \simeq B\left(\Lambda_{0}\right) \otimes B(W(\lambda))$.

The proof is similar to the one of Lemma 4.2.

\section{Proposition 4.6.}

(i) The vector $u_{\Lambda_{0}} \otimes u_{\lambda+n c_{k} \delta}$, regarded as a vector of $M_{n} / M_{n+1}$, is an extremal vector.

(ii)

$$
\xi_{0} \equiv \begin{cases}\Lambda_{0}+\lambda & \text { if }\left\langle h_{0}, \lambda\right\rangle=-1, \text { i.e. } a_{k}^{\vee}=1 \text { or } \mathfrak{g}=A_{2 n}^{(2)}, \\ \iota^{-1}\left(\Lambda_{0}\right) & \text { if } a_{0}^{\vee}=1, \frac{\left(\alpha_{k}, \alpha_{k}\right)}{2} \geqslant 1, \text { and } \iota \text { is the Dynkin } \\ & \text { diagram automorphism such that } t(\lambda) \in W \iota, \\ \Lambda_{4} & \text { if } \mathfrak{g}=F_{4}^{(1)} \text { and } k=3,\end{cases}
$$

$\bmod \mathbb{Q} \delta$. For the last case, see Figure 2 in the proof.

Proof. We may assume that $n=0$. If $u_{\Lambda_{0}} \otimes u_{\lambda} \in M_{1}$, then $u_{\lambda} \in$ $U_{q}^{+}(\mathfrak{g}) z_{k} u_{\mu}$, which is a contradiction. Hence $u_{\Lambda_{0}} \otimes u_{\lambda} \bmod M_{1}$ is a non-zero vector.

We divide the proof into three cases.

Case 1) $\quad a_{k}^{\vee}=1$ or $\mathfrak{g}=A_{2 n}^{(2)}$

In this case $\left\langle h_{0}, \lambda\right\rangle=-1$ and $c_{k}=1$ by Lemma 4.3. We shall show that $u_{\Lambda_{0}} \otimes u_{\lambda}$ is a highest weight vector of $M_{0} / M_{1}$. We have $e_{i}\left(u_{\Lambda_{0}} \otimes u_{\lambda}\right)=0$ for 
$i \neq 0$. We have $e_{0}\left(u_{\Lambda_{0}} \otimes u_{\lambda}\right)=u_{\Lambda_{0}} \otimes e_{0} u_{\lambda}$, and $e_{0} u_{\lambda}=S_{0} u_{\lambda}=u_{s_{\alpha_{0}-\delta} \lambda+\delta}=$ $z_{k} u_{s_{\alpha_{0}-\delta} \lambda} \in z_{k} U_{q}^{+}(\mathfrak{g}) u_{\mu}$.

Case 2) $\quad a_{0}^{\vee}=1$ and $\frac{\left(\alpha_{k}, \alpha_{k}\right)}{2} \geqslant 1$

We have $\lambda \in \widetilde{P}$ and hence $t(\lambda) \in \widetilde{W}$. We have then $t(-\lambda)\left(\Lambda_{0}+\lambda\right) \equiv \Lambda_{0}$ $\bmod \mathbb{Z} \delta$. Hence if we write $t(\lambda)=w \iota$ with $w \in W$ and a Dynkin diagram automorphism $\iota$, then we have $\xi_{0}=w^{-1}\left(\Lambda_{0}+\lambda\right)=\iota^{-1} t(-\lambda)\left(\Lambda_{0}+\lambda\right) \equiv \iota^{-1}\left(\Lambda_{0}\right)$ $\bmod \mathbb{Q} \delta$.

Set $v=S_{t(\lambda)}^{-1}\left(u_{\Lambda_{0}} \otimes u_{\lambda}\right)$. Here we regard $S_{\iota^{-1}}$ as an isomorphism $V\left(\Lambda_{0}\right) \otimes$ $V(\lambda) \rightarrow V\left(\iota^{-1} \Lambda_{0}\right) \otimes V\left(\iota^{-1} \lambda\right)$ such that $S_{\iota^{-1}}(a u)=\iota^{-1}(a) S_{\iota^{-1}}(u)$ for $a \in U_{q}^{-}(\mathfrak{g})$ and $u \in V\left(\Lambda_{0}\right) \otimes V(\lambda)$. Hence, $v$ is regarded as a vector in $V\left(\iota^{-1} \Lambda_{0}\right) \otimes V\left(\iota^{-1} \lambda\right)$. We shall show that $e_{i} v \in S_{\iota^{-1}} M_{1}$ for every $i \in I$.

(i) $i \neq 0, k$

In this case $t(\lambda) \alpha_{i}=\alpha_{i}$ holds. Hence we have $T_{t(\lambda)} e_{i}=e_{i}$, and

$$
S_{t(\lambda)} e_{i} v=\left(T_{t(\lambda)} e_{i}\right)\left(u_{\Lambda_{0}} \otimes u_{\lambda}\right)=e_{i}\left(u_{\Lambda_{0}} \otimes u_{\lambda}\right)=0 .
$$

(ii) $i=k$

Since $\left\langle h_{k}, \operatorname{wt}(v)\right\rangle=0$, it is enough to show that $e_{k} S_{k}^{-1} S_{t(\lambda)}^{-1}\left(u_{\Lambda_{0}} \otimes u_{\lambda}\right) \in$ $S_{\iota^{-1}} M_{1}$. This is equivalent to saying that $\left(T_{t(\lambda) s_{k}} e_{k}\right)\left(u_{\Lambda_{0}} \otimes u_{\lambda}\right) \in M_{1}$. Since $t(\lambda) s_{k} \alpha_{k}=c_{k} \delta-\alpha_{k} \in \Delta^{+}$, we have $T_{t(\lambda) s_{k}} e_{k} \in U_{q}^{+}(\mathfrak{g})$ and $\left(T_{t(\lambda) s_{k}} e_{k}\right)\left(u_{\Lambda_{0}} \otimes\right.$ $\left.u_{\lambda}\right)=u_{\Lambda_{0}} \otimes\left(T_{t(\lambda) s_{k}} e_{k}\right) u_{\lambda}$. The last factor is calculated as $\left(T_{t(\lambda) s_{k}} e_{k}\right) u_{\lambda}=$ $S_{t(\lambda) s_{k}} e_{k} S_{t(\lambda) s_{k}}^{-1} u_{\lambda}=S_{t(\lambda) s_{k}} e_{k} u_{s_{k} t(-\lambda) \lambda}$ up to a non-zero constant multiple. Since we have $\left\langle h_{k}, s_{k} t(-\lambda) \lambda\right\rangle=-\left\langle h_{k}, \lambda\right\rangle=-1$, we obtain $e_{k} u_{s_{k} t(-\lambda) \lambda}=$ $u_{t(-\lambda) \lambda}$. Thus we obtain $\left(T_{t(\lambda) s_{k}} e_{k}\right) u_{\lambda}=S_{t(\lambda) s_{k}} u_{t(-\lambda) \lambda}=u_{t(\lambda) s_{k} t(-\lambda) \lambda}$ up to a non-zero constant multiple. Since $t(\lambda) s_{k} t(-\lambda) \lambda=s_{t(\lambda) \alpha_{k}} \lambda=$ $s_{\alpha_{k}-c_{k} \delta} \lambda=s_{k} \lambda+c_{k} \delta$, we have

$$
u_{t(\lambda) s_{k} t(-\lambda) \lambda}=u_{s_{k} \lambda+c_{k} \delta}=z_{k} u_{s_{k} \lambda} \in z_{k} U_{q}^{+}(\mathfrak{g}) u_{\mu} .
$$

(iii) $i=0$

Let us first show that

$v={ }_{t(w \lambda)}^{-1}\left(u_{\Lambda_{0}} \otimes u_{w \lambda}\right)$ up to a non-zero constant multiple for every $w \in W_{0}$, by the induction of the length of $w$. Assuming that the assertion is true, we shall show that it is true for $s_{i} w$ for $i \in I_{0}$. According that $s_{i} t(w \lambda) \gtrless t(w \lambda)$, we have $S_{t\left(s_{i} w \lambda\right)}=S_{i}^{ \pm} S_{t(w \lambda)} S_{i}^{\mp}$. Hence we have

$$
\begin{aligned}
S_{t\left(s_{i} w \lambda\right)}^{-1}\left(u_{\Lambda_{0}} \otimes u_{s_{i} w \lambda}\right) & =S_{i}^{ \pm} S_{t(w \lambda)}^{-1} S_{i}^{\mp}\left(u_{\Lambda_{0}} \otimes u_{s_{i} w \lambda}\right) \\
& =S_{i}^{ \pm} S_{t(w \lambda)}^{-1}\left(u_{\Lambda_{0}} \otimes u_{w \lambda}\right)=S_{i}^{ \pm} v=v
\end{aligned}
$$


up to a non-zero constant multiple.

Now we divide the proof into two cases.

(a) $\left(\alpha_{k}, \alpha_{k}\right) / 2=1$

In this case, $c_{k}=1$. There exists $w \in W_{0}$ such that

$$
w^{-1} \alpha_{0} \equiv-\alpha_{k} \quad \bmod \mathbb{Z} \delta .
$$

Hence we have $\left\langle h_{0}, w \lambda\right\rangle=-1$, which implies that $t(w \lambda) \alpha_{0}=\alpha_{0}+\delta$ and $\left(t(w \lambda) \alpha_{0}, w \lambda\right)=-1$. Then we have

$$
\begin{aligned}
S_{t(w \lambda)} e_{0} v & =S_{t(w \lambda)} e_{0} S_{t(w \lambda)}^{-1}\left(u_{\Lambda_{0}} \otimes u_{w \lambda}\right) \\
& =u_{\Lambda_{0}} \otimes S_{t(w \lambda)} e_{0} S_{t(w \lambda)}^{-1} u_{w \lambda} \\
& =u_{\Lambda_{0}} \otimes S_{t(w \lambda)} S_{0} S_{t(w \lambda)}^{-1} u_{w \lambda} \\
& =u_{\Lambda_{0}} \otimes S_{\alpha_{0}+\delta} u_{w \lambda} \\
& =u_{\Lambda_{0}} \otimes u_{s_{\alpha_{0}+\delta} w \lambda} .
\end{aligned}
$$

On the other hand, we have

$$
\begin{aligned}
s_{\alpha_{0}+\delta} w \lambda & =w \lambda-\left(\alpha_{0}+\delta, w \lambda\right)\left(\alpha_{0}+\delta\right) \\
& =w \lambda-\left(\alpha_{0}-\delta, w \lambda\right)\left(\alpha_{0}-\delta\right)+2 \delta \\
& =s_{\delta-\alpha_{0}} w \lambda+2 \delta .
\end{aligned}
$$

This implies that

$$
u_{s_{\alpha_{0}+\delta} w \lambda}=z_{k}^{2} u_{s_{\delta-\alpha_{0}} w \lambda} \in z_{k} U_{q}^{+}(\mathfrak{g}) u_{\mu} .
$$

Hence $e_{0}\left(u_{\Lambda_{0}} \otimes u_{\lambda}\right) \in S_{t(w \lambda)}^{-1} M_{1}=S_{\iota^{-1}} M_{1}$.

(b) $\left(\alpha_{k}, \alpha_{k}\right) / 2>1$

In this case, by the classification of affine Dynkin diagrams, there exists $i \neq 0, k$ such that $\left(\alpha_{i}, \alpha_{i}\right) / 2=1$. Let us take $w \in W_{0}$ such that $w^{-1} \alpha_{0} \equiv \alpha_{i} \bmod \mathbb{Z} \delta$. Hence $\left(w^{-1} \alpha_{0}, \lambda\right)=0$, which implies $t(w \lambda) \alpha_{0}=\alpha_{0}$. Hence we have $S_{t(w \lambda)} e_{0} S_{t(w \lambda)}^{-1}=e_{0}$ and

$$
\begin{aligned}
S_{t(w \lambda)} e_{0} v & =S_{t(w \lambda)} e_{0} S_{t(w \lambda)}^{-1}\left(u_{\Lambda_{0}} \otimes u_{w \lambda}\right) \\
& =u_{\Lambda_{0}} \otimes e_{0} u_{w \lambda} .
\end{aligned}
$$

Since $\left(\alpha_{0}, w \lambda\right)=\left(w^{-1} \alpha_{0}, \lambda\right)=0, e_{0} u_{w \lambda}$ must vanish. 
Case 3) the remaining case (i.e. $a_{0}^{\vee}=1, a_{k}^{\vee}>1$ and $\left(\alpha_{k}, \alpha_{k}\right) / 2<1$ )

By the classification of affine Dynkin diagrams, there is only one remaining case, namely $\mathfrak{g}=F_{4}^{(1)}$ and $k=3$ :

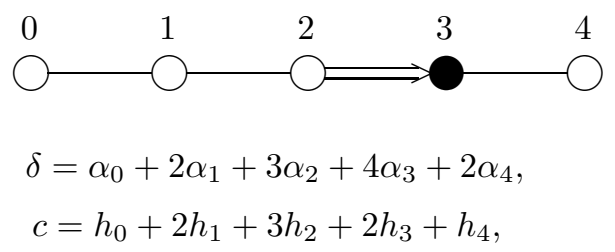

Figure 2. $\mathfrak{g}=F_{4}^{(1)}$

We have in this case $c_{3}=1, \lambda=\Lambda_{3}-2 \Lambda_{0}$ and $\mu=\lambda-4 \alpha_{1}-8 \alpha_{2}-12 \alpha_{3}-$ $6 \alpha_{4}$.

We have

$s_{4} s_{3} s_{2} s_{1} s_{0}\left(\Lambda_{0}+\lambda\right)=\Lambda_{0}+\lambda+\alpha_{0}+\alpha_{1}+\alpha_{2}+\alpha_{3}+\alpha_{4} \equiv \Lambda_{4} \quad \bmod \mathbb{Z} \delta$.

Set $x=s_{0} s_{1} s_{2} s_{3} s_{4}$ and $v=S_{x}^{-1}\left(u_{\Lambda_{0}} \otimes u_{\lambda}\right)$. Let us show that $v$ is a highest weight vector of $M_{0} / M_{1}$, i.e. $e_{i} v \in M_{1}$ for $i \in I$.

(1) $i=0$

Since $x \alpha_{0}=\alpha_{1}$, we have $S_{x} e_{0} v=\left(T_{x} e_{0}\right)\left(u_{\Lambda_{0}} \otimes u_{\lambda}\right)=e_{1}\left(u_{\Lambda_{0}} \otimes u_{\lambda}\right)=0$.

(2) $i=1$

Since $x \alpha_{1}=\alpha_{2}$, we can conclude $e_{1} v=0$ by the same argument as above.

(3) $i=2$

We have $x \alpha_{2}=\alpha_{0}+\alpha_{1}+\alpha_{2}+2 \alpha_{3}$. Hence we have

$$
\begin{aligned}
S_{x} e_{2} v & =S_{x} e_{2} S_{x}^{-1}\left(u_{\Lambda_{0}} \otimes u_{\lambda}\right) \\
& =u_{\Lambda_{0}} \otimes\left(S_{x} e_{2} S_{x}^{-1} u_{\lambda}\right),
\end{aligned}
$$

and since $\left\langle x h_{2}, \lambda\right\rangle=-1$, we have

$$
\begin{aligned}
S_{x} e_{2} S_{x}^{-1} u_{\lambda} & =S_{x} S_{2} S_{x}^{-1} u_{\lambda}=u_{s_{x \alpha_{2}} \lambda} \\
& =u_{\lambda+\alpha_{0}+\alpha_{1}+\alpha_{2}+2 \alpha_{3}} \\
& =u_{\lambda+\delta-\alpha_{1}-2 \alpha_{2}-2 \alpha_{3}-2 \alpha_{4}} \\
& =z_{k} u_{\lambda-\alpha_{1}-2 \alpha_{2}-2 \alpha_{3}-2 \alpha_{4}} \in z_{k} U_{q}^{+}(\mathfrak{g}) u_{\mu} .
\end{aligned}
$$

This implies that $e_{2} v \in M_{1}$. 
(4) $i=3$

$e_{3} v=0$ follows from $x \alpha_{3}=\alpha_{4}$.

(5) $i=4$

We have seen that $v \in M_{0} / M_{1}$ is invariant by $S_{2}$ and $S_{3}$. Hence it is enough to show that $e_{4} S_{3}^{-1} S_{2}^{-1} v=0$. Since $x s_{2} s_{3} \alpha_{4}=\alpha_{3}$, we have

$$
S_{x s_{2} s_{3}} e_{4} S_{3}^{-1} S_{2}^{-1} v=\left(T_{x s_{2} s_{3}} e_{4}\right)\left(u_{\Lambda_{0}} \otimes u_{\lambda}\right)=e_{3}\left(u_{\Lambda_{0}} \otimes u_{\lambda}\right)=0 .
$$

\section{Theorem 4.7.}

$$
U_{q}^{-}(\mathfrak{g})\left(u_{\Lambda_{0}} \otimes u_{\lambda}\right) / U_{q}^{-}(\mathfrak{g})\left(u_{\Lambda_{0}} \otimes u_{\lambda+c_{k} \delta}\right) \simeq V\left(\Lambda_{0}+\lambda\right) .
$$

By this isomorphism, $u_{\Lambda_{0}} \otimes u_{\lambda}$ corresponds to $u_{\Lambda_{0}+\lambda}$.

Proof. By the preceding proposition, there exists a morphism $g: V\left(\Lambda_{0}+\right.$ $\lambda) \rightarrow M_{0} / M_{1}$, sending $u_{\Lambda_{0}+\lambda}$ to $u_{\Lambda_{0}} \otimes u_{\lambda} \bmod M_{1}$. On the other hand, Theorem 3.3 implies the existence of a morphism $\psi: M_{0} \rightarrow V\left(\Lambda_{0}+\lambda\right)$. Since $\Lambda_{0}+\lambda+c_{k} \delta$ is not a weight of $V\left(\Lambda_{0}+\lambda\right), \psi$ factors through $M_{0} / M_{1}$ and thus we obtain a morphism $M_{0} / M_{1} \rightarrow V\left(\Lambda_{0}+\lambda\right)$ sending $u_{\Lambda_{0}} \otimes u_{\lambda}$ to $u_{\Lambda_{0}+\lambda}$. Obviously it is an inverse of $g$.

Note that the theorem holds if we replace $\lambda$ with $\mu$.

Corollary 4.8. $\quad u_{\Lambda_{0}} \otimes\left(B^{+}(\mu) \backslash B^{+}\left(\mu+c_{k} \delta\right)\right) \simeq B^{+}\left(\Lambda_{0}+\mu\right)$. In particular $B(W(\lambda)) \simeq B^{+}\left(\Lambda_{0}+\mu\right)$ as a crystal over $\mathfrak{g}_{0}$.

Proof. By the preceding theorem, we have

$$
U_{q}^{+}(\mathfrak{g}) u_{\Lambda_{0}+\mu} \simeq U_{q}^{+}(\mathfrak{g})\left(u_{\Lambda_{0}} \otimes u_{\mu}\right) /\left(U_{q}^{-}(\mathfrak{g})\left(u_{\Lambda_{0}} \otimes u_{\mu+c_{k} \delta}\right) \cap U_{q}^{+}(\mathfrak{g})\left(u_{\Lambda_{0}} \otimes u_{\mu}\right)\right)
$$

On the other hand, Corollary 3.2 implies that $U_{q}^{-}(\mathfrak{g})\left(u_{\Lambda_{0}} \otimes u_{\mu+c_{k} \delta}\right) \cap U_{q}^{+}(\mathfrak{g})\left(u_{\Lambda_{0}} \otimes\right.$ $\left.u_{\mu}\right) \subset U_{q}^{-}(\mathfrak{g})\left(u_{\Lambda_{0}} \otimes u_{\mu+c_{k} \delta}\right) \cap u_{\Lambda_{0}} \otimes V(\mu)=u_{\Lambda_{0}} \otimes U_{q}^{+}(\mathfrak{g}) u_{\mu+c_{k} \delta}$, which implies that $U_{q}^{-}(\mathfrak{g})\left(u_{\Lambda_{0}} \otimes u_{\mu+c_{k} \delta}\right) \cap U_{q}^{+}(\mathfrak{g})\left(u_{\Lambda_{0}} \otimes u_{\mu}\right)=u_{\Lambda_{0}} \otimes U_{q}^{+}(\mathfrak{g}) u_{\mu+c_{k} \delta}$. Hence we have

$$
U_{q}^{+}(\mathfrak{g}) u_{\Lambda_{0}+\mu} \simeq\left(u_{\Lambda_{0}} \otimes U_{q}^{+}(\mathfrak{g}) u_{\mu}\right) /\left(u_{\Lambda_{0}} \otimes U_{q}^{+}(\mathfrak{g}) u_{\mu+c_{k} \delta}\right) .
$$

Thus we obtain the desired result.

Lemma 4.5 and Theorem 4.7 imply the following result. 
Corollary 4.9. $B\left(\Lambda_{0}\right) \otimes B(W(\lambda)) \simeq B\left(\Lambda_{0}+\lambda\right)$.

Corollary 4.10. There exists a unique vector $b \in B(W(\lambda))$ such that $\varepsilon_{i}(b) \leqslant \delta_{i, 0}$ for all $i$.

Proof. The condition is equivalent to saying that $u_{\Lambda_{0}} \otimes b$ is a highest weight vector, and the preceding corollary implies that $B\left(\Lambda_{0}\right) \otimes B(W(\lambda))$ has a unique highest weight vector.

\section{Corollary 4.11.}

(i) If $b \in B(\mu)$ satisfies $\operatorname{wt}(b) \notin \xi_{0}-\Lambda_{0}-c_{k} \delta+Q_{-}$, then $b \in B^{+}(\mu)$.

(ii) If an integral weight $\eta$ satisfies $\eta \notin \xi_{0}-\Lambda_{0}-c_{k} \delta+Q_{-}$, then $V(\lambda)_{\eta}=$ $\left(U_{q}^{+}(\mathfrak{g}) u_{\mu}\right)_{\eta}$.

Proof. There exist $b^{\prime} \in B^{+}(\mu) \backslash B\left(\mu+c_{k} \delta\right)$ and $n \in \mathbb{Z}$ such that $b=z_{k}^{n} b^{\prime}$. By Corollary 4.8, we have $\operatorname{wt}\left(u_{\Lambda_{0}} \otimes b^{\prime}\right)=\Lambda_{0}+\operatorname{wt}(b)-n c_{k} \delta \in \xi_{0}+Q_{-}$. Hence the assumption implies $n \geqslant 0$. Thus we conclude (i), and (ii) follows from (i).

\section{Acknowledgements}

The author thanks B. Feigin, M. Jimbo, T. Miwa, E. Mukhin, Y. Takeyama, and M. Okado for helpful discussions.

\section{References}

[1] Akasaka, T. and Kashiwara, M., Finite-dimensional representations of quantum affine algebras, Publ. RIMS, Kyoto Univ., 33 (1997), 839-867.

[2] Beck, J., Nakajima, H., Crystal bases and two-sided cells of quantum affine algebras, math. QA/0212253.

[3] Chari, Vyjayanthi, On the fermionic formula and the Kirillov-Reshetikhin conjecture, Int. Math. Res. Not., 12 (2001), 629-654.

[4] Hatayama, G., Kuniba, A., Okado, M., Takagi, T. and Yamada, Y., Remarks on fermionic formula, Contemp. Math., 248 (1999), 243-291.

[5] Hatayama, G., Kuniba, A., Okado, M., Takagi, T. and Tsuboi, Z., Paths, crystals and fermionic formulae, in "Math Phys Odyssey 2001-Integrable Models and Beyond In Hornor of Barry M. McCoy", Edited by M. Kashiwara and T. Miwa, Birkhäuser (2002), 205-272.

[6] Kuniba, A., Misra, K. C., Okado, M., Takagi, T. and Uchiyama, J., Crystals for Demazure modules of classical affine Lie algebras, J. Algebra, 208 (1998), 185-215.

[7] Kang, S.-J., Kashiwara, M., Misra, K., Miwa, T., Nakashima, T. and Nakayashiki, A., Affine crystals and Vertex models, Internat. J. Modern Phys. A, 7, Suppl. 1A (1992), 449-484. 
[8] Kang, S.-J., Kashiwara, M., Misra, K., Miwa, T., Nakashima, T. and Nakayashiki, A., Perfect crystals of quantum affine Lie algebra, Duke Math. J., 68 (1992) 499-607.

[9] Kashiwara, M., On crystal bases, Representations of Groups, Proceedings of the 1994 Annual Seminar of the Canadian Math. Soc. Banff Center, Banff, Alberta, June 15-24, (B.N. Allison and G.H. Cliff, eds), CMS Conf. Proc., 16 (1995) 155-197, Amer. Math. Soc., Providence, RI.

[10] , On crystal bases of the $q$-analogue of universal enveloping algebras, Duke Math. J., 63 (1991), 465-516.

[11] Global crystal bases of quantum groups, Duke Math. J., 69 (1993), 455-485.

[12] Crystal base and Littelmann's refined Demazure character formula, Duke Math. J., 71 (1993), 839-858.

[13] (1994), 383-413.

[14] (2002), 117-175.

[15] - Bases cristallines des groupes quantiques, Cours spécialisé 9, Société Mathémathique de France (noted by Charles Cochet) 2002.

[16] Kleber, M., Combinatorial structure of finite-dimensional representations of Yangians: the simply-laced case, Int. Math. Res. Not., 4 (1997), 187-201.

[17] Kirillov, A. N., Reshetikhin, N., Representation of Yangians and multiplicity of occurrence of the irreducible components of the tensor product of representations of simple Lie algebras, J. Sov. Math., 52 (1990), 3156-3164.

[18] Kuniba, A., Nakanishi, T., Suzuki, J., Functional relations in solvable lattice models. I, Functional relations and representation theory. Internat. J. Modern Phys. A, 9 (1994), 5215-5266.

[19] Lusztig, G., Introduction to Quantum Groups, Progr. Math., 110 (1993), Birkhäuser Boston, Boston, MA.

[20] - Quantum groups at $v=\infty$, Functional analysis on the eve of the 21st century, Vol. 1 (New Brunswick, NJ, 1993), Progr. Math., 131 (1995) 199-221, Birkhäuser Boston, Boston, MA.

[21] Nakajima, H., Extremal weight modules of quantum affine algebras, math.QA/0204183.

$[22] \_, t$-analogs of $q$-characters of Kirillov-Reshetikhin modules of quantum affine algebras, math.QA/0204185.

[23] Saito, Y., PBW basis of quantized universal enveloping algebras, Publ. RIMS, Kyoto Univ., 30 (1994), 209-232. 